\title{
Equiconvergence theorems for Chébli-Trimèche hypergroups
}

\author{
LUCA BRANDOLINI AND GIACOMO GIGANTE
}

\begin{abstract}
We consider a Sturm-Liouville operator of the kind $\frac{d^{2}}{d t^{2}}+\frac{A^{\prime}(t)}{A(t)} \frac{d}{d t}$ on $(0,+\infty)$ and the related eigenfunction expansion. We prove that, under suitable assumptions on $A(t)$, the partial sums of the Fourier integral associated to such expansion behave like the partial sums of the classical Fourier-Bessel transform. This implies an almost everywhere convergence result for $L^{p}(A(t) d t)$ functions. Our methods rely on asymptotic expansions for the eigenfunctions and the Harish-Chandra function that we prove under very weak hypotheses.
\end{abstract}

Mathematics Subject Classification (2000): 43A62 (primary); 43A32, 34L10 (secondary).

Differential operators of the kind

$$
\mathcal{L}=\frac{d^{2}}{d t^{2}}+\frac{A^{\prime}(t)}{A(t)} \frac{d}{d t}
$$

and the associated spectral decompositions arise naturally in harmonic analysis. For example when $A(t)=t^{n-1}$, the operator $\mathcal{L}$ is the radial part of the Laplacian in $\mathbb{R}^{n}$ and in this case the associated spectral decomposition is the so called FourierBessel expansion that corresponds to the harmonic analysis of radial functions in $\mathbb{R}^{n}$. This transform is defined for any $\alpha \geqslant-\frac{1}{2}$ by

$$
\mathcal{F}_{\alpha} f(\lambda)=\int_{0}^{+\infty} f(t) 2^{\alpha} \Gamma(\alpha+1) \frac{J_{\alpha}(\lambda t)}{(\lambda t)^{\alpha}} t^{2 \alpha+1} d t
$$

but of course it can be interpreted as a Fourier transform of a radial function only when $\alpha=(n-2) / 2$. The inversion formula associated to this transform is given by

$$
f(t)=\int_{0}^{+\infty} \mathcal{F}_{\alpha} f(\lambda) 2^{\alpha} \Gamma(\alpha+1) \frac{J_{\alpha}(\lambda t)}{(\lambda t)^{\alpha}} \frac{\lambda^{2 \alpha+1} d \lambda}{4^{\alpha} \Gamma^{2}(\alpha+1)} .
$$

In a non compact symmetric space of rank one there are values of $\alpha$ and $\beta$ such that, setting $A(t)=(\sinh t)^{2 \alpha+1}(\cosh t)^{2 \beta+1}$, one obtains the radial part of the Laplace-Beltrami operator and in this case the associated spectral decomposition is

Received March 17, 2008; accepted September 3, 2008. 
the harmonic analysis of spherical functions. More precisely in this setting one has the Fourier-Jacobi transform

$$
\mathcal{H}_{\alpha, \beta} f(\lambda)=\int_{0}^{+\infty} f(t) \varphi_{\lambda}(t)(\sinh t)^{2 \alpha+1}(\cosh t)^{2 \beta+1} d t
$$

and the inversion formula

$$
f(t)=\int_{0}^{+\infty} \mathcal{H}_{\alpha, \beta} f(\lambda) \varphi_{\lambda}(t) \frac{d \lambda}{2 \pi|c(\lambda)|^{2}}
$$

where

$$
\varphi_{\lambda}(t)={ }_{2} F_{1}\left(\frac{\alpha+\beta+1-i \lambda}{2}, \frac{\alpha+\beta+1+i \lambda}{2} ; \alpha+1 ;-\sinh ^{2}(t)\right)
$$

is the Jacobi function (see e.g. [6] or [8]) and

$$
c(\lambda)=\frac{\Gamma(\alpha+1) \Gamma(i \lambda / 2) \Gamma\left(\frac{(1+i \lambda)}{2}\right)}{2 \sqrt{\pi} \Gamma\left(\frac{\alpha+\beta+1+i \lambda}{2}\right) \Gamma\left(\frac{\alpha-\beta+1+i \lambda}{2}\right)}
$$

is the Harish-Chandra function.

In this paper we will study the convergence properties of the inversion of the expansion associated to the operator $\mathcal{L}$, under suitable assumption on the function $A(t)$.

Our starting point is a theorem of Colzani, Crespi, Travaglini and Vignati (see [6]) that can be stated in the following way.

Let $\alpha \geqslant-\frac{1}{2}$ and let

$$
T_{R}^{\alpha} f(t)=\int_{0}^{R} \mathcal{F}_{\alpha} f(\lambda) 2^{\alpha} \Gamma(\alpha+1) \frac{J_{\alpha}(\lambda t)}{(\lambda t)^{\alpha}} \frac{\lambda^{2 \alpha+1} d \lambda}{4^{\alpha} \Gamma^{2}(\alpha+1)}
$$

be the $R$-th partial sum of the Fourier-Bessel integral. When $\alpha=-\frac{1}{2}$ we obtain the classical cosine expansion and in this case we set $C_{R}=T_{R}^{-\frac{1}{2}}$.

Theorem 0.1. Let $f \in L^{1}\left(\mathbb{R}^{+}, \frac{t^{\alpha+\frac{1}{2}}}{1+t} d t\right)$ and let $0<t<+\infty$, then

$$
\lim _{R \rightarrow+\infty}\left|T_{R}^{\alpha} f(t)-t^{-\alpha-\frac{1}{2}} C_{R}\left[(\cdot)^{\alpha+\frac{1}{2}} f(\cdot)\right](t)\right|=0 .
$$

Moreover the convergence is uniform in every interval $0<\varepsilon<t<\eta<+\infty$. 
In [6] the authors also sketch a similar equiconvergence result for the FourierJacobi expansion $\mathcal{H}_{\alpha, \beta}$ in the case $\alpha=\frac{1}{2}$ and $\beta=\frac{1}{2}$.

One of the main results of this paper is an equiconvergence theorem for the partial sums associated to the spectral decomposition of operator of the kind $\mathcal{L}=\frac{d^{2}}{d t^{2}}+$ $\frac{A^{\prime}(t)}{A(t)} \frac{d}{d t}$. More precisely we will show that such partial sums behave like the partial sums of the Fourier-Bessel transform for a suitable value of the dimensional parameter $\alpha$.

\subsection{Assumptions}

We now introduce the main hypotheses on the function $A(t)$.

Let $A:[0,+\infty) \rightarrow \mathbb{R}$ and assume that

(H1) $A \in \mathcal{C}^{\infty}(0,+\infty)$ and it is continuous in 0 .

(H2) $A$ is positive in $(0,+\infty)$.

(H3) There exists $\alpha>-\frac{1}{2}$ and an odd function $B \in \mathcal{C}^{\infty}(\mathbb{R})$ such that

$$
\frac{A^{\prime}(t)}{A(t)}=\frac{2 \alpha+1}{t}+B(t) \quad \text { for } t \in(0,+\infty) .
$$

(H4) $\frac{A^{\prime}}{A}$ is nonnegative, decreasing on $(0,+\infty)$ and $\lim _{t \rightarrow+\infty} A(t)=+\infty$.

By (H3) we have $A(t) \sim c t^{2 \alpha+1}$ as $t \rightarrow 0^{+}$. From now on we assume that $A(t)$ is normalized in such a way that $c=1$. We also set

$$
2 \rho=\lim _{t \rightarrow+\infty} \frac{A^{\prime}(t)}{A(t)} .
$$

The above assumptions allow to define in the half-line the so called Chébli-Trimèche hypergroup (see [1, Section 3.5] for the details). The harmonic analysis associated to the differential operator $\mathcal{L}$ and the corresponding hypergroup have been studied by several authors. We refer the reader to [1] and [16].

Let $\lambda \in \mathbb{C}$, by Bôcher [3] the Cauchy problem

$$
\left\{\begin{array}{l}
\mathcal{L} u+\left(\lambda^{2}+\rho^{2}\right) u=0 \\
u(0)=1 \\
u^{\prime}(0)=0
\end{array}\right.
$$

has a unique solution $\varphi_{\lambda}$ defined in $[0,+\infty)$. It is an easy task to see that $\left|\varphi_{\lambda}(x)\right| \leqslant$ 1 for $\lambda^{2}+\rho^{2} \geqslant 0$. Indeed, multiplying by $\varphi_{\lambda}^{\prime}$ the above equation and integrating one obtains

$$
\int_{0}^{x} \varphi_{\lambda}^{\prime} \mathcal{L} \varphi_{\lambda} d t+\left(\lambda^{2}+\rho^{2}\right) \int_{0}^{x} \varphi_{\lambda} \varphi_{\lambda}^{\prime} d t=0
$$


and a direct computation shows that the first term is positive and the second one equals $\frac{1}{2}\left(\lambda^{2}+\rho^{2}\right)\left[\varphi_{\lambda}(x)^{2}-\varphi_{\lambda}(0)^{2}\right]$. It follows that $\left|\varphi_{\lambda}(x)\right| \leqslant 1$.

Let now $f \in L^{1}(A(t) d t)$. The Fourier transform of $f$ is defined by

$$
\widehat{f}(\lambda)=\int_{0}^{+\infty} f(t) \varphi_{\lambda}(t) A(t) d t .
$$

For this Fourier transform an inversion formula is given by

$$
f(t)=\int_{0}^{+\infty} \widehat{f}(\lambda) \varphi_{\lambda}(t) \frac{d \lambda}{2 \pi|c(\lambda)|^{2}}
$$

where $c(\lambda)$ is associated the Harish-Chandra function (see Section 1.3 below).

By means of the classical Liouville transformation

$$
v(t)=\sqrt{A(t)} u(t)
$$

the equation $\mathcal{L} u+\left(\lambda^{2}+\rho^{2}\right) u=0$ can be written as

$$
v^{\prime \prime}+\lambda^{2} v=q(t) v
$$

where

$$
q(t)=\frac{1}{4}\left(\frac{A^{\prime}(t)}{A(t)}\right)^{2}+\frac{1}{2}\left(\frac{A^{\prime}(t)}{A(t)}\right)^{\prime}-\rho^{2} .
$$

Since $\frac{A^{\prime}(t)}{A(t)}=\frac{2 \alpha+1}{t}+B(t)$ by (H3) the above equation takes the form

$$
v^{\prime \prime}+\left(\lambda^{2}-\frac{\alpha^{2}-\frac{1}{4}}{t^{2}}\right) v=G(t) v
$$

where

$$
G(t)=\frac{1}{2} B^{\prime}(t)+\left(\alpha+\frac{1}{2}\right) \frac{B(t)}{t}+\frac{B(t)^{2}}{4}-\rho^{2} .
$$

Being $G(t)$ smooth, equation (0.5) can be seen as a perturbation of Bessel equation and one can expect that its solutions can be approximated at the origin by Bessel functions. This is indeed the case, at least for large $\lambda$, as we will show in Theorem 1.2. To deal with the case $\lambda$ small we need more precise information on the behavior of $q(t)$ at infinity. We make the following extra assumption.

(H5) There exists $a \geqslant 0$ such that

$$
q(t)=\frac{a^{2}-1 / 4}{t^{2}}+\zeta(t)
$$

for some $\zeta \in L^{1}((1,+\infty), t d t)$ for $a>0$ or $\zeta \in L^{1}((1,+\infty), t \log t d t)$ for $a=0$. 
We will show in Remark 1.15 that under the above assumption for $t \rightarrow+\infty$

$$
\sqrt{A(t)} \varphi_{0}(t) \sim c t^{\frac{1}{2}+b}
$$

where $b>-\frac{1}{2}$ and $|b|=a$ or

$$
\sqrt{A(t)} \varphi_{0}(t) \sim c t^{\frac{1}{2}} \log t .
$$

This last singular case may occur only for $a=0$. In this case we set $b=0$.

In some cases we need a stronger integrability condition on the function $\zeta(t)$. Namely

(H6) When $-\frac{1}{2}<b<0$ and $\sqrt{A(t)} \varphi_{0}(t) \sim c t^{\frac{1}{2}+b}$ we assume that $\zeta(t) \in$ $L^{1}\left((1,+\infty), t^{2|b|+1} d t\right)$. When $b=0$ and $\sqrt{A(t)} \varphi_{0}(t) \sim c t^{\frac{1}{2}}$ we assume $\zeta(t) \in L^{1}\left((1,+\infty), t \log ^{2} t d t\right)$.

We will show that this case can be given only when $\rho=0$, so that $\varphi_{0}(t) \equiv 1$.

\subsection{Main results}

In order to state our main results let us define the following partial sum operators

$$
S_{R} f(t)=\int_{0}^{R} \widehat{f}(\lambda) \varphi_{\lambda}(t) \frac{d \lambda}{2 \pi|c(\lambda)|^{2}},
$$

and

$$
F_{R} f(t)=\frac{t^{\alpha+\frac{1}{2}}}{\sqrt{A(t)}} T_{R}^{\alpha}\left(\frac{\sqrt{A}}{(\cdot)^{\alpha+\frac{1}{2}}} f\right)(t) .
$$

Our main result is the following

Theorem 0.2. Assume (H1) to (H6) hold and let $\zeta^{\prime}(t) \in L^{1}((1,+\infty), d t)$. Then for every $f \in L^{1}\left(\mathbb{R}^{+}, \frac{\sqrt{A(t)}}{1+t} d t\right), S_{R} f(t)$ and $F_{R} f(t)$ are well defined and for every $t \in[0,+\infty)$

$$
\lim _{R \rightarrow+\infty}\left|S_{R} f(t)-F_{R} f(t)\right|=0 .
$$

Corollary 0.3. Assume (H1) to (H6) hold and let $\zeta^{\prime}(t) \in L^{1}((1,+\infty), d t)$. Assume $\rho=0$ and $\frac{4 \alpha+4}{2 \alpha+3}<q<\frac{4 b+4}{2 b+1}$, or $\rho>0$ and $\frac{4 \alpha+4}{2 \alpha+3}<q \leqslant 2$. Then for every $f \in L^{q}\left(\mathbb{R}^{+}, A(t) d t\right)$

$$
\lim _{R \rightarrow+\infty} S_{R} f(t)=f(t) \text { a.e. }
$$


In the case of noncompact symmetric spaces of rank one, the above Corollary was obtained by Meaney and Prestini in [12] and [13], using the boundedness of the related maximal operator.

The next Theorem shows that the above results are essentially sharp.

Theorem 0.4. Assume (H1) to (H5) hold. If $\rho>0$ the operator $S_{R}$ cannot be defined from $L^{p}\left(\mathbb{R}^{+}, A(t) d t\right)$ into the space of tempered distributions for any $p>$ 2. If $\rho=0$ the operator $S_{R}$ cannot be defined from $L^{p}\left(\mathbb{R}^{+}, A(t) d t\right)$ into the space of tempered distributions for any $p \geqslant \frac{4 b+4}{2 b+1}$.

Theorem 0.5. Assume (H1) to (H5) hold. There exists $f \in L^{\frac{4 \alpha+4}{2 \alpha+3}}\left(\mathbb{R}^{+}, A(t) d t\right)$ supported in $(0,1)$ such that $S_{R} f(x)$ diverges as $R \rightarrow+\infty$ for every $x \in(0,+\infty)$.

As we pointed out before, the equiconvergence Theorem 0.2 is based upon the asymptotic expansions of the eigenfunctions $\varphi_{\lambda}(x)$ and the Harish-Chandra function $c(\lambda)$. Some of this kind of results are well known, some are not. In particular, our techniques require to know the behaviour of the function $c(\lambda)$ for $\operatorname{Im} \lambda \leqslant 0$ and $|\lambda|$ small. In [2], Bloom and $\mathrm{Xu}$ solve this problem, apart from the case which, in our notation, corresponds to $\rho=0,-1 / 2<b \leqslant 0, A(t)$ recessive (see Definition 1.11). This case has been studied by O. Bracco in his Ph. D. thesis [4], but only in the case $\lambda \in \mathbb{R}$. In this paper we completely solve the problem, with weaker hypotheses than those required in [2] (see Theorem 2.4 here). In an effort to make the paper more accessible, even for the reader unfamiliar with this subject, we decided to state all these approximation results, both old and new, with complete proofs. Sections 1 and 2 are therefore devoted to these matters, and are essentially selfcontained. In Section 3 we prove the convergence and divergence results. In the Appendix we state some general results on asymptotic approximation of eigenvalue problems, that we use repeatedly in Sections 1 and 2.

As a final remark, we would like to emphasize that the proof of the equiconvergence result does not require the Fourier inversion formula (0.2). This, in fact, is an immediate consequence of Corollary 0.3.

Corollary 0.6 (Inversion formula (see [2])). Assume (H1) to (H6) hold and let $\zeta^{\prime}(t) \in L^{1}((1,+\infty), d t)$. Let $f \in L^{1}\left(\mathbb{R}^{+}, A(t) d t\right) \cap \mathcal{C}\left(\mathbb{R}^{+}\right)$and assume that $\widehat{f} \in L^{1}\left(\mathbb{R}^{+}, \frac{d \lambda}{2 \pi|c(\lambda)|^{2}}\right)$, then

$$
f(t)=\int_{0}^{+\infty} \widehat{f}(\lambda) \varphi_{\lambda}(t) \frac{d \lambda}{2 \pi|c(\lambda)|^{2}} .
$$

\subsection{Notation and preliminary estimates}

In this section we introduce the notation that will be used throughout the paper. We will also recall some definitions and well known estimates for Bessel functions. 
By $f(t) \sim g(t)$ as $t \rightarrow t_{0}$ we mean

$$
\lim _{t \rightarrow t_{0}} \frac{f(t)}{g(t)}=1 .
$$

By $f(t)=o(g(t))$ as $t \rightarrow t_{0}$ we mean

$$
\lim _{t \rightarrow t_{0}} \frac{f(t)}{g(t)}=0 .
$$

By $f(t)=O(g(t))$ we mean that there is a positive constant $c$ such that

$$
\left|\frac{f(t)}{g(t)}\right| \leqslant c .
$$

By $f(t) \approx g(t)$ we mean that there are positive constants $c_{1}$ and $c_{2}$ such that

$$
c_{1} \leqslant \frac{f(t)}{g(t)} \leqslant c_{2} .
$$

The symbol $\mathbb{C}^{*}$ denotes the non zero complex numbers, $\mathbb{R}^{+}$denotes the positive real numbers and $\mathbb{R}^{-}$denotes the negative real numbers.

The Wronskian determinant of the functions $f$ and $g$ is the function $\mathcal{W}(f, g)=$ $f g^{\prime}-f^{\prime} g$.

The Bessel function of the first kind of order $v$ will be as usual denoted by $J_{v}(x)$, the Bessel function of the second kind of order $v$ by $Y_{v}(x)$ and the Bessel functions of third kind (Hankel functions) of order $v$ by $H_{v}^{(1)}(x)$ and $H_{v}^{(2)}(x)$,

$$
\begin{aligned}
& H_{v}^{(1)}(x)=J_{v}(x)+i Y_{v}(x) \\
& H_{v}^{(2)}(x)=J_{v}(x)-i Y_{v}(x) .
\end{aligned}
$$

We will use the notation

$$
\begin{gathered}
\mathcal{J}_{v}(x)=\sqrt{x} J_{v}(x) \\
\mathcal{Y}_{v}(x)=\sqrt{x} Y_{v}(x) \\
\mathcal{H}_{v}^{(1)}(x)=\sqrt{x} H_{v}^{(1)}(x) \\
\mathcal{H}_{v}^{(2)}(x)=\sqrt{x} H_{v}^{(2)}(x) .
\end{gathered}
$$

All these are multivalued holomorphic functions on $\mathbb{C}^{*}$, and we will consider their principal branch on the complex plane cut along the ray $(-\infty, 0]$. For a negative $x$ we will agree to define

$$
\mathcal{H}_{v}^{(2)}(x)=\lim _{\substack{z \rightarrow x \\ \operatorname{Im} \mathbf{z}<0}} \mathcal{H}_{v}^{(2)}(z)
$$


In other words we will take the principal branch of $\mathcal{H}_{v}^{(2)}(z)$ for all $z \in \mathbb{C}^{*}$ with $-\pi \leqslant \arg (z)<\pi$.

In the sequel we will use the notation $\langle x\rangle=\frac{|x|}{1+|x|}$. Observe that for small value of $x,\langle x\rangle \approx|x|$ while for large $x,\langle x\rangle \approx 1$.

Proposition 0.7. For all $v>-\frac{1}{2}$ the following estimates hold uniformly in $t>0$, $\lambda \in \mathbb{C}^{*}$.

$$
\begin{gathered}
\left|\mathcal{J}_{\nu}(\lambda t)\right| \leqslant C\langle\lambda t\rangle^{\nu+1 / 2} e^{|\operatorname{Im} \lambda| t}, \\
\left|\frac{\partial}{\partial t}\left(\mathcal{J}_{\nu}(\lambda t)\right)\right| \leqslant C|\lambda|\langle\lambda t\rangle^{\nu-1 / 2} e^{|\operatorname{Im} \lambda| t} .
\end{gathered}
$$

For $v \neq 0$,

$$
\begin{aligned}
\left|\mathcal{Y}_{\nu}(\lambda t)\right| & \leqslant C\langle\lambda t\rangle^{-|\nu|+1 / 2} e^{|\operatorname{Im} \lambda| t}, \\
\left|\frac{\partial}{\partial t}\left(\mathcal{Y}_{\nu}(\lambda t)\right)\right| & \leqslant C|\lambda|\langle\lambda t\rangle^{-|\nu|-1 / 2} e^{|\operatorname{Im} \lambda| t} .
\end{aligned}
$$

Also

$$
\begin{gathered}
\left|\mathcal{Y}_{0}(\lambda t)\right| \leqslant C\langle\lambda t\rangle^{1 / 2} \log \left(\frac{2}{\langle\lambda t\rangle}\right) e^{|\operatorname{Im} \lambda| t}, \\
\left|\frac{\partial}{\partial t}\left(\mathcal{Y}_{0}(\lambda t)\right)\right| \leqslant C|\lambda|\langle\lambda t\rangle^{-1 / 2} \log \left(\frac{2}{\langle\lambda t\rangle}\right) e^{|\operatorname{Im} \lambda| t} .
\end{gathered}
$$

For $v \neq 0$,

$$
\begin{aligned}
\left|\mathcal{H}_{v}^{(2)}(\lambda t)\right| & \leqslant C\langle\lambda t\rangle^{-v+1 / 2} e^{\operatorname{Im} \lambda t}, \\
\left|\frac{\partial}{\partial t}\left(\mathcal{H}_{v}^{(2)}(\lambda t)\right)\right| & \leqslant C|\lambda|\langle\lambda t\rangle^{-v-1 / 2} e^{\operatorname{Im} \lambda t} .
\end{aligned}
$$

Also

$$
\begin{gathered}
\left|\mathcal{H}_{0}^{(2)}(\lambda t)\right| \leqslant C\langle\lambda t\rangle^{1 / 2} \log \left(\frac{2}{\langle\lambda t\rangle}\right) e^{\operatorname{Im} \lambda t}, \\
\left|\frac{\partial}{\partial t}\left(\mathcal{H}_{0}^{(2)}(\lambda t)\right)\right|
\end{gathered}
$$

See $[10$, Chapter 5] for the details. 


\section{Asymptotic expansion of the eigenfunctions}

\subsection{Estimates for $\varphi_{\lambda}$ for large $\lambda$}

In [7] Fitouhi and Hamza obtained an asymptotic expansion of the kind

$$
\sqrt{A(t)} \varphi_{\lambda}(t)=\sum_{m=0}^{M} a_{m}(t) \frac{\mathcal{J}_{\alpha+m}(\lambda t)}{\lambda^{m+\alpha+1 / 2}}+R_{M}(\lambda, t)
$$

and suitable estimate for the remainder valid for $\lambda \in \mathbb{R}$ and $t>0$. This expansion essentially quantifies the effect of the perturbation $B(t)$ of assumption (H3) by showing that the eigenfunction $\varphi_{\lambda}$ can be approximated using Bessel functions. In the case of noncompact symmetric spaces this expansion has been previously obtained by Stanton and Tomas (see [14]).

For the proof of Theorem 0.2 we need estimates for the remainder valid for $\lambda \in \mathbb{C}$. This estimates will be proved in Theorem 1.2 using a technique similar to that of Fitouhi and Hamza.

Lemma 1.1. Let $G$ be a smooth even function. Define recursively

$$
\begin{aligned}
b_{0} & =2^{\alpha} \Gamma(\alpha+1) \\
b_{m+1}(t) & =-\frac{1}{2 t^{m+1}} \int_{0}^{t} s^{m}\left(b_{m}^{\prime \prime}(s)+\frac{b_{m}^{\prime}(s)}{s}(1-2 \alpha)-G(s) b_{m}(s)\right) d s .
\end{aligned}
$$

Then $b_{m}$ is smooth and even. Also, calling $a_{m}(t)=t^{m} b_{m}(t)$ we have

$$
a_{m}^{\prime \prime}+\frac{1-2 \alpha-2 m}{t} a_{m}^{\prime}+\frac{2 \alpha m+m^{2}}{t^{2}} a_{m}-G(t) a_{m}+2 a_{m+1}^{\prime}=0 .
$$

Furthermore, assume that for all $0 \leqslant k \leqslant M$ one has

$$
G^{(k)} \in L^{1}((1,+\infty), d t) .
$$

Then for every $0 \leqslant m \leqslant M+1$ and $1 \leqslant k \leqslant M-m+2$ one has

$$
a_{m}^{(k)} \in L^{1}((1,+\infty), d t) .
$$

Proof. Assume by induction that $b_{m}$ is smooth and even, then

$$
s^{m}\left(b_{m}^{\prime \prime}(s)+\frac{b_{m}^{\prime}(s)}{s}(1-2 \alpha)-G(s) b_{m}(s)\right)
$$

has the parity of $m$, it is smooth and is $O\left(s^{m}\right)$ near zero. Therefore its integral has the parity of $m+1$ and is $O\left(t^{m+1}\right)$ near zero. Multiplication by $t^{-m-1}$ gives an even smooth function. Equation (1.1) follows directly from the recursive definition of $b_{m+1}$. 
Assume now

$$
G^{(k)} \in L^{1}((1,+\infty), d t)
$$

for all $0 \leqslant k \leqslant M$. To prove (1.2) assume by induction (on $m$ ) that $a_{m}^{(k)} \in L^{1}$ for $1 \leqslant k \leqslant M-m+2$.

Observe that since

$$
\begin{aligned}
a_{m+1}(t) & =-\frac{1}{2} \int_{0}^{t}\left(s^{m} b_{m}^{\prime \prime}+s^{m-1} b_{m}^{\prime}(1-2 \alpha)-G(s) a_{m}\right) d s \\
& =-\frac{1}{2} \int_{0}^{t} a_{m}^{\prime \prime}+\frac{1-2 \alpha-2 m}{s} a_{m}^{\prime}+a_{m}\left(\frac{m^{2}+2 \alpha m}{s^{2}}-G(s)\right) d s
\end{aligned}
$$

$a_{m+1}^{(k)}$ is a linear combination of expressions of the form $t^{-k-1+\ell} a_{m}^{(\ell)}$ for $\ell=0, \ldots, k+$ 1 or $G^{(k-1-\ell)} a_{m}^{(\ell)}$ for $\ell=0, \ldots k-1$, all integrable by the induction hypothesis if $1 \leqslant k \leqslant M-m+1$.

Theorem 1.2. Assume that (H1) to (H4) hold. Let $G$ as in (0.6), let $M \geqslant 0$ and assume $G^{(k)} \in L^{1}((1,+\infty), d t)$ for $0 \leqslant k \leqslant M$. Then for $\lambda \in \mathbb{C}^{*}$ and $t \geqslant 0$

$$
\sqrt{A(t)} \varphi_{\lambda}(t)=\sum_{m=0}^{M} a_{m}(t) \frac{\mathcal{J}_{\alpha+m}(\lambda t)}{\lambda^{m+\alpha+1 / 2}}+R_{M}(\lambda, t)
$$

where the coefficients $a_{m}$ are as in Lemma 1.1 and the remainder satisfies the following estimates. If $\alpha \neq 0$

$$
\left|R_{M}(\lambda, t)\right| \leqslant C \frac{\langle\lambda t\rangle^{\alpha+M+\frac{3}{2}}\langle t\rangle^{M+1}}{|\lambda|^{\alpha+3 / 2+M}} e^{|\operatorname{Im}(\lambda)| t} \Theta(t)
$$

and

$$
\left|\frac{\partial}{\partial t} R_{M}(\lambda, t)\right| \leqslant C \frac{\langle\lambda t\rangle^{\alpha+M+\frac{1}{2}}\langle t\rangle^{M+1}}{|\lambda|^{\alpha+1 / 2+M}} e^{|\operatorname{Im}(\lambda)| t} \Theta(t)
$$

where $\Theta(t)=e^{\frac{C}{|\lambda|} \int_{0}^{t}|G(v)| d v}$. If $\alpha=0$

$$
\left|R_{M}(\lambda, t)\right| \leqslant C \frac{\langle\lambda t\rangle^{M+\frac{3}{2}}\langle t\rangle^{M+1} \log \frac{2}{\langle\lambda t\rangle}}{|\lambda|^{3 / 2+M}} e^{|\operatorname{Im}(\lambda)| t} \Theta(t)
$$

and

$$
\left|\frac{\partial}{\partial t} R_{M}(\lambda, t)\right| \leqslant C \frac{\langle\lambda t\rangle^{M+\frac{1}{2}}\langle t\rangle^{M+1} \log \frac{2}{\langle\lambda t\rangle}}{|\lambda|^{1 / 2+M}} e^{|\operatorname{Im}(\lambda)| t} \Theta(t) .
$$


Proof. Observe that, $\varphi_{\lambda} \equiv \varphi_{-\lambda}$ and that, being $\frac{\mathcal{J}_{\alpha+m}(\lambda t)}{\lambda^{m+\alpha+1 / 2}}$ entire and even in $\lambda$, the above expansion only needs to be proved for $\lambda \in \mathbb{C}^{*} \backslash \mathbb{R}^{-}$. Replacing the expression

$$
\sum_{m=0}^{M} a_{m}(t) \frac{\mathcal{J}_{\alpha+m}(\lambda t)}{\lambda^{m+\alpha+1 / 2}}+R_{M}(\lambda, t)
$$

in equation (0.5) we obtain

$$
\begin{gathered}
\frac{1}{\lambda^{\alpha+1 / 2}} \sum_{m=0}^{M}\left\{\frac{a_{m}^{\prime \prime}}{\lambda^{m}} \mathcal{J}_{\alpha+m}(\lambda t)+2 \frac{a_{m}^{\prime}}{\lambda^{m-1}} \mathcal{J}_{\alpha+m}^{\prime}(\lambda t)+\frac{a_{m}}{\lambda^{m-2}} \mathcal{J}_{\alpha+m}^{\prime \prime}(\lambda t)\right. \\
\left.+\left(\lambda^{2}-\frac{\alpha^{2}-\frac{1}{4}}{t^{2}}\right) \frac{a_{m}}{\lambda^{m}} \mathcal{J}_{\alpha+m}(\lambda t)-G(t) \frac{a_{m}}{\lambda^{m}} \mathcal{J}_{\alpha+m}(\lambda t)\right\} \\
+\frac{d^{2}}{d t^{2}} R_{M}(\lambda, t)+\left(\lambda^{2}-\frac{\alpha^{2}-\frac{1}{4}}{t^{2}}-G(t)\right) R_{M}(\lambda, t)=0
\end{gathered}
$$

Using the well known identities

$$
\begin{aligned}
& \mathcal{J}_{\alpha}^{\prime}(t)=\frac{\left(\frac{1}{2}-\alpha\right)}{t} \mathcal{J}_{\alpha}(t)+\mathcal{J}_{\alpha-1}(t) \\
& \mathcal{J}_{\alpha}^{\prime \prime}(t)=\left(\frac{\alpha^{2}-\frac{1}{4}}{t^{2}}-1\right) \mathcal{J}_{\alpha}(t),
\end{aligned}
$$

and the recurrence relation defining the coefficients $a_{m}(t)$, we obtain

$$
\frac{d^{2} R_{M}}{d t^{2}}+\left(\lambda^{2}-\frac{\alpha^{2}-\frac{1}{4}}{t^{2}}\right) R_{M}=G(t) R_{M}+2 a_{M+1}^{\prime} \frac{\mathcal{J}_{\alpha+M}(\lambda t)}{\lambda^{M+\alpha+1 / 2}}
$$

The associated homogeneous equation

$$
v^{\prime \prime}+\left(\lambda^{2}-\frac{\alpha^{2}-\frac{1}{4}}{t^{2}}\right) v=0
$$


has the linearly independent solutions $\mathcal{J}_{\alpha}(\lambda t)$ and $\mathcal{Y}_{\alpha}(\lambda t)$, with Wronskian

$$
\mathcal{W}\left(\mathcal{J}_{\alpha}(\lambda t), \mathcal{Y}_{\alpha}(\lambda t)\right)=\frac{2 \lambda}{\pi}
$$

(see [10, Section 5.9]). In view of Lemma A.1, a solution of the integral equation

$$
\begin{aligned}
R_{M}(\lambda, t)= & -\pi \int_{0}^{t} \frac{\mathcal{J}_{\alpha}(\lambda t) \mathcal{Y}_{\alpha}(\lambda s)-\mathcal{Y}_{\alpha}(\lambda t) \mathcal{J}_{\alpha}(\lambda s)}{2 \lambda} \\
& \times\left\{G(s) R_{M}(\lambda, s)+2 a_{M+1}^{\prime}(s) \frac{\mathcal{J}_{\alpha+M}(\lambda s)}{\lambda^{M+\alpha+1 / 2}}\right\} d s,
\end{aligned}
$$

also satisfies equation (1.3). We will clarify later that there is a solution of the above integral equation which satisfies the required Cauchy conditions, and therefore there is no ambiguity in calling $R_{M}(\lambda, s)$ the solution of (1.4). We now apply Theorem A.2 with kernel

$$
k(t, s)=-\pi \frac{\mathcal{J}_{\alpha}(\lambda t) \mathcal{Y}_{\alpha}(\lambda s)-\mathcal{Y}_{\alpha}(\lambda t) \mathcal{J}_{\alpha}(\lambda s)}{2 \lambda} .
$$

In order to estimate the above kernel we point out that it can also be represented in the following forms

$$
\begin{aligned}
& k(t, s)=-\pi \frac{\mathcal{J}_{-\alpha}(\lambda t) \mathcal{J}_{\alpha}(\lambda s)-\mathcal{J}_{\alpha}(\lambda t) \mathcal{J}_{-\alpha}(\lambda s)}{2 \lambda \sin (\pi \alpha)} \\
& k(t, s)=-\pi i \frac{\mathcal{H}_{\alpha}^{(1)}(\lambda t) \mathcal{H}_{\alpha}^{(2)}(\lambda s)-\mathcal{H}_{\alpha}^{(1)}(\lambda s) \mathcal{H}_{\alpha}^{(2)}(\lambda t)}{4 \lambda} .
\end{aligned}
$$

Classical estimates on Bessel and Hankel functions applied to (1.5) when $|\lambda t| \leqslant 1$ and $\alpha \geqslant 0$, to (1.6) when $|\lambda t| \leqslant 1$ and $-\frac{1}{2}<\alpha<0$ and to (1.7) when $|\lambda t|>1$ give (see in [4, Lemma 1.5]), for $0<s \leqslant t$,

$$
\begin{array}{r}
|k(t, s)| \leqslant \begin{cases}C \frac{1}{|\lambda|}\langle\lambda t\rangle^{|\alpha|+1 / 2} e^{|\operatorname{Im}(\lambda)|(t-s)}\langle\lambda s\rangle^{-|\alpha|+1 / 2}, & \text { if } \alpha \neq 0 \\
C \frac{1}{|\lambda|}\langle\lambda t\rangle^{1 / 2} e^{|\operatorname{Im}(\lambda)|(t-s)}\langle\lambda s\rangle^{1 / 2} \log \frac{2}{\langle\lambda s\rangle}, & \text { if } \alpha=0\end{cases} \\
\left|\frac{\partial k}{\partial t}(t, s)\right| \leqslant \begin{cases}C\langle\lambda t\rangle^{|\alpha|-1 / 2} e^{|\operatorname{Im}(\lambda)|(t-s)}\langle\lambda s\rangle^{-|\alpha|+1 / 2}, & \text { if } \alpha \neq 0 \\
C\langle\lambda t\rangle^{-1 / 2} e^{|\operatorname{Im}(\lambda)|(t-s)}\langle\lambda s\rangle^{1 / 2} \log \frac{2}{\langle\lambda s\rangle}, & \text { if } \alpha=0\end{cases}
\end{array}
$$


and the hypotheses of Theorem A.2 are satisfied with

$$
\begin{aligned}
& P_{0}(t)=C \frac{1}{|\lambda|}\langle\lambda t\rangle^{|\alpha|+1 / 2} e^{|\operatorname{Im}(\lambda)| t} \\
& P_{1}(t)=C\langle\lambda t\rangle^{|\alpha|-1 / 2} e^{|\operatorname{Im}(\lambda)| t} \\
& Q(s)= \begin{cases}\langle\lambda s\rangle^{-|\alpha|+1 / 2} e^{-|\operatorname{Im}(\lambda)| s}, & \text { if } \alpha \neq 0 \\
\langle\lambda s\rangle^{1 / 2} \log \left(\frac{2}{\langle\lambda s\rangle}\right) e^{-|\operatorname{Im}(\lambda)| s}, & \text { if } \alpha=0\end{cases} \\
& \phi(s)=2 a_{M+1}^{\prime}(s) \frac{\mathcal{J}_{\alpha+M}(\lambda s)}{\lambda^{M+\alpha+1 / 2}} Q(s) \\
& J(s)=Q(s)^{-1} .
\end{aligned}
$$

Observe that

$$
\kappa_{0}=\sup _{t>0} P_{0}(t) Q(t)=\frac{C}{|\lambda|}
$$

and

$$
\kappa=\sup _{t>0} Q(t)|J(t)|=1 .
$$

The integral

$$
\Phi(t)=\int_{0}^{t}|\phi(s)| d s
$$

converges, since

$$
|\phi(s)| \leqslant \begin{cases}C|\lambda|^{-\alpha-1 / 2-M}\left|a_{M+1}^{\prime}(s)\right|\langle\lambda s\rangle^{\alpha-|\alpha|+M+1}, & \text { if } \alpha \neq 0 \\ C|\lambda|^{-1 / 2-M}\left|a_{M+1}^{\prime}(s)\right|\langle\lambda s\rangle^{M+1} \log \left(\frac{2}{\langle\lambda t\rangle}\right), & \text { if } \alpha=0 .\end{cases}
$$

Actually, noticing that

$$
\left|a_{M+1}^{\prime}(s)\right| \leqslant C s^{M}
$$

for $s<1$ and that $a_{M+1}^{\prime} \in L^{1}((1,+\infty), d t)$ a simple computation shows that

$$
\Phi(t) \leqslant \begin{cases}C|\lambda|^{-\alpha-1 / 2-M}\langle\lambda t\rangle^{\alpha-|\alpha|+M+1}\langle t\rangle^{M+1} & \text { for } \alpha \neq 0 \\ C|\lambda|^{-1 / 2-M}\langle\lambda t\rangle^{M+1}\langle t\rangle^{M+1} \log \frac{2}{\langle\lambda t\rangle} & \text { for } \alpha=0 .\end{cases}
$$

Therefore there is a continuously differentiable solution $R_{M}(\lambda, t)$ satisfying the estimates given in the statement of Theorem A.2.

Since $\sqrt{A(t)}=t^{\alpha+1 / 2}(1+o(t))$ as $t \rightarrow 0^{+}$(by (H3)) the initial conditions

$$
\varphi_{\lambda}(0)=1, \quad \varphi_{\lambda}^{\prime}(0)=0
$$


become after the Liouville transformation

$$
\sqrt{A(t)} \varphi_{\lambda}(t)=t^{\alpha+1 / 2}(1+o(t)) \text { as } t \rightarrow 0 .
$$

The expression

$$
\sum_{m=0}^{M} \frac{a_{m}(t)}{\lambda^{m+\alpha+1 / 2}} \mathcal{J}_{\alpha+m}(\lambda t)+R_{M}(\lambda, t)
$$

trivially satisfies this condition, as

$$
\sum_{m=0}^{M} \frac{a_{m}(t)}{\lambda^{m+\alpha+1 / 2}} \mathcal{J}_{\alpha+m}(\lambda t)=t^{\alpha+1 / 2}+o\left(t^{\alpha+3 / 2}\right)
$$

while $\left|R_{M}(\lambda, t)\right| \leqslant C_{\lambda} t^{\alpha+5 / 2}\left|\log \left(\frac{|\lambda| t}{4}\right)\right|=o\left(t^{\alpha+3 / 2}\right)$ as $t \rightarrow 0$.

Remark 1.3. The quantity $\frac{C}{|\lambda|}$ that appears in $\Theta(t)$ blows up as $\lambda \rightarrow 0$. It is not difficult to improve such estimate in order to solve this problem. Indeed, in the above proof $\kappa_{0}$ has been computed assuming that $t \in[0,+\infty)$. Taking $t \in[0, \eta]$ gives $\kappa_{0}=\sup _{t \in[0, \eta]} P_{0}(t) Q(t)=\frac{C \eta}{1+\eta|\lambda|}$ and therefore $\Theta(t)=e^{\frac{C \eta}{1+\eta|\lambda|} \int_{0}^{t}|G(v)| d v}$. Since, for every given $t \in(0,+\infty)$, we can take $\eta=t$, we obtain

$$
\Theta(t)=e^{\frac{C t}{1+t|\lambda|} \int_{0}^{t}|G(v)| d v} .
$$

\subsection{Estimates for $\varphi_{\lambda}$ for small $\lambda$}

In this section we will show that the equation $v^{\prime \prime}=q v$ that is satisfied by $\sqrt{A(t)} \varphi_{0}(t)$, has two independent solutions $W_{1}$ and $W_{2}$ that have different behavior at infinity. Namely $W_{1}(t) \approx t^{-a+\frac{1}{2}}$ and $W_{2}(t) \approx t^{a+\frac{1}{2}}$. Consequently $\sqrt{A(t)} \varphi_{0}(t)$ has one of these two possible behaviors. In the following we will study under which conditions these two behaviors occur. This will play a key role in the study of the HarishChandra function as we will see in Chapter 2.

In the next Lemma we obtain a bound from below for $\varphi_{i \eta}(t)$ when $-\rho \leqslant \eta \leqslant 0$.

Lemma 1.4. Assume (H1) to (H4) hold and let $-\rho \leqslant \eta<0$. Then

$$
\varphi_{i \eta}(t) \geqslant e^{-(\rho+\eta) t}
$$

Also

$$
\varphi_{0}(t) \geqslant e^{-\rho t}(1+\rho t) .
$$

Remark 1.5. The first estimate of the above Lemma follows readily from the Laplace representation of $\varphi_{i \eta}$ (see e.g. [5]). The second estimate seems to be new. We give a direct proof of both. 
Proof. When $\eta=-\rho$ the estimate is trivial since $\varphi_{-i \rho} \equiv 1$. Assume $\rho>0$ and $-\rho<\eta \leqslant 0$ and let $g(t)=e^{(\rho+\eta) t} \varphi_{i \eta}(t)$. A simple computation shows that $g$ satisfies the problem

$$
\left\{\begin{array}{l}
g^{\prime \prime}+\left(\frac{A^{\prime}(t)}{A(t)}-2(\rho+\eta)\right) g^{\prime}-(\rho+\eta)\left(\frac{A^{\prime}(t)}{A(t)}-2 \rho\right) g=0 \\
g(0)=1 \\
g^{\prime}(0)=\rho+\eta
\end{array}\right.
$$

Consider first $\eta=0$. We only need to show that $g^{\prime}(t) \geqslant \rho$ for every $t \geqslant 0$. By contradiction assume $g^{\prime}(t)<\rho$ for some $t$ and let

$$
t_{0}=\inf \left\{t \geqslant 0: g^{\prime}(t)<\rho\right\} .
$$

Our assumptions imply that $\varphi_{0}^{\prime \prime}(0)=-\frac{\rho^{2}}{2 \alpha+2}$ so that $g^{\prime \prime}(0)=\frac{2 \alpha+1}{2 \alpha+2} \rho^{2}>0$, since $g^{\prime}(0)=\rho$ we have $t_{0}>0$. Also $g^{\prime}\left(t_{0}\right)=\rho$ and $g^{\prime \prime}\left(t_{0}\right) \leqslant 0$. Being $g^{\prime}(t) \geqslant \rho$ on $\left[0, t_{0}\right]$ we have $g\left(t_{0}\right) \geqslant 1+\rho t_{0}$. By the definition of $t_{0}$ there exists $t_{1}>t_{0}$ such that $g^{\prime \prime}\left(t_{1}\right)<0, g^{\prime}\left(t_{1}\right)<\rho$ and $g\left(t_{1}\right) \geqslant 1$.

Substituting into the differential equations yields

$$
g^{\prime \prime}\left(t_{1}\right)+\left(\frac{A^{\prime}\left(t_{1}\right)}{A\left(t_{1}\right)}-2 \rho\right)\left(g^{\prime}\left(t_{1}\right)-\rho g\left(t_{1}\right)\right)=0
$$

which contradicts $g^{\prime \prime}\left(t_{1}\right)<0$.

Let now $-\rho<\eta<0$. In this case it is enough to show that $g^{\prime}(t) \geqslant 0$ for every $t \geqslant 0$. By contradiction assume $g^{\prime}(t)<0$ for some $t$ and let

$$
t_{0}=\inf \left\{t \geqslant 0: g^{\prime}(t)<0\right\} \text {. }
$$

Since $g^{\prime}(0)=\rho+\eta>0$ we have $t_{0}>0$ so that $g^{\prime \prime}\left(t_{0}\right) \leqslant 0$. As in the previous case there exists $t_{1}>t_{0}$ such that $g^{\prime \prime}\left(t_{1}\right)<0, g^{\prime}\left(t_{1}\right)<0$ and $g\left(t_{1}\right)>1$. Substituting into the differential equation we obtain

$$
g^{\prime \prime}\left(t_{1}\right)+\left(\frac{A^{\prime}\left(t_{1}\right)}{A\left(t_{1}\right)}-2(\rho+\eta)\right) g^{\prime}\left(t_{1}\right)=(\rho+\eta)\left(\frac{A^{\prime}\left(t_{1}\right)}{A\left(t_{1}\right)}-2 \rho\right) g\left(t_{1}\right)
$$

that contradicts $g^{\prime \prime}\left(t_{1}\right)<0$.

Remark 1.6. A close look at the proof shows that $\varphi_{i \eta}(t)=e^{-\rho t} g(t)$ where $g$ is a increasing function for $-\rho<\eta<0$ and $g^{\prime}(t) \geqslant \rho$ for $\eta=0$. In both cases $g(0)=1$.

The following lemma shows that under our assumptions, $\rho>0$ implies $a \geqslant \frac{1}{2}$. This fact will be useful in the sequel.

Lemma 1.7. Let assumptions $(\mathrm{H} 1)$ to $(\mathrm{H} 4)$ be satisfied and let $\rho>0$. Then $(\mathrm{H} 5)$ cannot hold for any $a<\frac{1}{2}$. 
Proof. Assume, by contradiction that (H5) holds for some $0 \leqslant a<\frac{1}{2}$. Let

$$
\frac{A^{\prime}(t)}{A(t)}=2 \rho+h(t)
$$

by (0.4) and assumption (H5) we have

$$
q(t)=\rho h(t)+\frac{1}{4} h(t)^{2}+\frac{1}{2} h^{\prime}(t)=\frac{a^{2}-\frac{1}{4}}{t^{2}}+\zeta(t)
$$

where $\zeta \in L^{1}((1,+\infty), t d t)$. Then

$$
\rho h(t)+\frac{1}{2} h^{\prime}(t) \leqslant \rho h(t)+\frac{1}{4} h(t)^{2}+\frac{1}{2} h^{\prime}(t)=\frac{a^{2}-\frac{1}{4}}{t^{2}}+\zeta(t),
$$

so that

$$
2 \rho t h(t)+t h^{\prime}(t) \leqslant 2 \frac{a^{2}-\frac{1}{4}}{t}+2 t \zeta(t)
$$

Therefore

$$
\int_{z}^{t}\left(2 \rho s h(s)+s h^{\prime}(s)\right) d s \leqslant 2\left(a^{2}-\frac{1}{4}\right) \log \frac{t}{z}+2 \int_{z}^{+\infty} s|\zeta(s)| d s .
$$

Integrating by parts we obtain

$$
\operatorname{th}(t)-z h(z)+\int_{z}^{t}(2 \rho s-1) h(s) d s \leqslant 2\left(a^{2}-\frac{1}{4}\right) \log \frac{t}{z}+2 \int_{z}^{+\infty} s|\zeta(s)| d s .
$$

Taking $z>\frac{1}{2 \rho}$ we have

$$
-z h(z) \leqslant 2\left(a^{2}-\frac{1}{4}\right) \log \frac{t}{z}+2 \int_{z}^{+\infty} s|\zeta(s)| d s
$$

and letting $t \rightarrow+\infty$ we get a contradiction.

We now consider the behavior of $\varphi_{0}(t)$ as $t \rightarrow+\infty$. We will show that under assumption (H5) the differential equation $\mathcal{L} u+\rho^{2} u=0$, satisfied by $\varphi_{0}(t)$ has two linearly independent solutions that behave like $A(t)^{-\frac{1}{2}} t^{ \pm a+\frac{1}{2}}$ when $a>0$ and like $A(t)^{-\frac{1}{2}} t^{\frac{1}{2}}$ and $A(t)^{-\frac{1}{2}} t^{\frac{1}{2}} \log t$ when $a=0$.

The next result can be found in the proof of [2, Proposition 3.17]. See also [4]. 
Theorem 1.8. Assume (H1) to (H5) hold and let

$$
k(t, s)=\frac{t^{-a+\frac{1}{2}} s^{a+\frac{1}{2}}-t^{a+\frac{1}{2}} s^{-a+\frac{1}{2}}}{2 a} \zeta(s)
$$

when $a>0$ or

$$
k(t, s)=\left(t^{\frac{1}{2}} s^{\frac{1}{2}} \log (s)-t^{\frac{1}{2}} \log (t) s^{\frac{1}{2}}\right) \zeta(s)
$$

when $a=0$. Then the equation

$$
v^{\prime \prime}=q v
$$

has a unique solution $W_{1}$ that satisfies the integral equation

$$
W_{1}(t)=t^{-a+\frac{1}{2}}+\int_{t}^{+\infty} k(t, s) W_{1}(s) d s
$$

and such that, setting

$$
W_{1}(t)=t^{-a+\frac{1}{2}}+E(t),
$$

the error term $E(t)$ satisfies

$$
\begin{aligned}
& |E(t)| \leqslant t^{-a+\frac{1}{2}}\left(e^{c \int_{t}^{+\infty} s|\zeta(s)| d s}-1\right), \\
& \left|E^{\prime}(t)\right| \leqslant t^{-a-\frac{1}{2}}\left(e^{c \int_{t}^{+\infty} s|\zeta(s)| d s}-1\right)
\end{aligned}
$$

for $a>0$ and

$$
\begin{array}{r}
|E(t)| \leqslant t^{\frac{1}{2}}\left(e^{c \int_{t}^{+\infty} s \log (1+s)|\zeta(s)| d s}-1\right), \\
\left|E^{\prime}(t)\right| \leqslant t^{-\frac{1}{2}}\left(e^{c \int_{t}^{+\infty} s \log (1+s)|\zeta(s)| d s}-1\right)
\end{array}
$$

for $a=0$.

Proof. We write the equation (1.9) in the form

$$
v^{\prime \prime}-\frac{a^{2}-\frac{1}{4}}{t^{2}} v=\zeta(t) v
$$

and we observe that the associated homogeneous equation has for $a>0$ the solutions $t^{a+\frac{1}{2}}$ and $t^{-a+\frac{1}{2}}$ while for $a=0$ the solutions are $t^{\frac{1}{2}}$ and $t^{\frac{1}{2}} \log t$. The fact that a solution $W_{1}(t)$ of (1.10) is also a solution of (1.9) is a consequence of Lemma A.1. The existence and uniqueness of $W_{1}$ satisfying the estimates for $E(t)=W_{1}(t)-t^{-a+\frac{1}{2}}$ follow from Theorem A.2. 
Corollary 1.9. Assume (H1) to (H5) hold. Then the equation

$$
v^{\prime \prime}=q v
$$

has a solution $V$ satisfying $V(t) \approx t^{a+\frac{1}{2}}$ as $t \rightarrow+\infty$ for $a>0$ and $V(t) \approx$ $t^{\frac{1}{2}} \log (t)$ as $t \rightarrow+\infty$ for $a=0$.

Proof. Let $t_{0}$ be large enough and let

$$
V(t)=\int_{t_{0}}^{t} \frac{W_{1}(t)}{\left(W_{1}(s)\right)^{2}} d s
$$

It is not difficult to check that $V$ satisfies (1.11) and the required estimates.

Remark 1.10. Since $\sqrt{A} \varphi_{0}$ is a solution of equation (1.11) we have $\sqrt{A} \varphi_{0}=$ $c_{1} W_{1}(t)+c_{2} V(t)$. If follows that $\sqrt{A} \varphi_{0}$ has two possible behaviors at infinity. If $c_{2} \neq 0$ we have $\sqrt{A} \varphi_{0} \approx V(t)$ otherwise $\sqrt{A} \varphi_{0}=c_{1} W_{1}(t)$.

In order to distinguish these two possible behaviors we introduce the following terminology.

Definition 1.11. Assume (H1) to (H5) hold for some $a \geqslant 0$. If $\sqrt{A} \varphi_{0}(t)=$ $c_{1} W_{1}(t)$ for some $c_{1} \neq 0$ we say that $A(t)$ is recessive. Otherwise we say that $A(t)$ is dominant.

Lemma 1.12. Assume (H1) to (H5) hold with $\rho>0$ then $A(t)$ is dominant.

Proof. By Lemma 1.7 we must have $a \geqslant \frac{1}{2}$. By contradiction assume that $A(t)$ is recessive. By $(\mathrm{H} 3)$ and $(0.3)$ we have $\sqrt{A(t)} \geqslant c e^{\rho t}$ for large $t$. Applying Lemma 1.4 we have

$$
C e^{\rho t}(1+\rho t) e^{-\rho t} \leqslant \sqrt{A(t)} \varphi_{0}(t)=c W_{1}(t) \approx t^{-a+\frac{1}{2}}
$$

that letting $t \rightarrow+\infty$ gives a contradiction.

Lemma 1.13. Assume (H1) to (H5) hold with $\rho=0$ and $a \geqslant \frac{1}{2}$ then $A(t)$ is dominant.

Proof. Assume by contradiction that $A(t)$ is recessive. When $\rho=0$ we have $\varphi_{0}(t) \equiv 1$. Therefore

$$
\sqrt{A(t)}=c W_{1}(t) \approx t^{-a+\frac{1}{2}}
$$

that contradicts the fact that $A(t) \rightarrow+\infty$ as $t \rightarrow+\infty$.

Lemma 1.14. Assume (H1) to (H5) hold with $\rho=0$ and $0 \leqslant a<\frac{1}{2}$ then $A(t)$ is dominant if and only is $\frac{A(t)}{t}$ is unbounded as $t \rightarrow+\infty$. 
Proof. Assume $A(t)$ is recessive, then $\sqrt{A(t)}=c W_{1}(t) \approx t^{-a+\frac{1}{2}}$ so that $\frac{A(t)}{t} \approx$ $t^{-2 a}$. If instead $A(t)$ is dominant then $\sqrt{A(t)} \approx t^{\frac{1}{2}+a}$ for $a>0$ or $\sqrt{A(t)} \approx$ $t^{\frac{1}{2}} \log t$.

Remark 1.15. We have shown that, if (H1) to (H5) hold, there is a $b>-1 / 2$, $|b|=a$, such that $\sqrt{A(t)} \varphi_{0}(t) \approx t^{\frac{1}{2}+b}$, except when $A$ is dominant and $a=0$, in which case $\sqrt{A(t)} \varphi_{0}(t) \approx t^{\frac{1}{2}} \log t$. In the former case the values $-\frac{1}{2}<b \leqslant 0$ correspond to $A(t)$ recessive while the values $b>0$ correspond to $A(t)$ dominant. In the latter case we will say that $A$ is singular.

To deal with the recessive case we need a solution of equation (1.9) constructed in a way similar to the one used to construct $W_{1}$. This requires the stronger integrability condition on $q$ described in assumption (H6).

Theorem 1.16. Assume (H1) to (H6) hold with $A(t)$ recessive. Let $k(t, s)$ as in Theorem 1.8. For $-\frac{1}{2}<b<0$ the equation

$$
v^{\prime \prime}=q v
$$

has a unique solution $W_{2}(t)$ that satisfies the integral equation

$$
W_{2}(t)=t^{a+\frac{1}{2}}+\int_{t}^{+\infty} k(t, s) W_{2}(s) d s,
$$

such that, setting $W_{2}(t)=t^{a+\frac{1}{2}}+E(t)$, we have

$$
\begin{aligned}
& |E(t)| \leqslant t^{-a+\frac{1}{2}}\left(e^{c \int_{t}^{+\infty} s^{2 a+1}|\zeta(s)| d s}-1\right), \\
& \left|E^{\prime}(t)\right| \leqslant t^{-a-\frac{1}{2}}\left(e^{c \int_{t}^{+\infty} s^{2 a+1}|\zeta(s)| d s}-1\right) .
\end{aligned}
$$

When $b=0$ the above equation has a unique solution $W_{2}(t)$ satisfying the integral equation

$$
W_{2}(t)=t^{\frac{1}{2}} \log (t)+\int_{t}^{+\infty} k(t, s) W_{2}(s) d s
$$

such that, setting $W_{2}(t)=t^{\frac{1}{2}} \log (t)+E(t)$ we have

$$
\begin{gathered}
|E(t)| \leqslant t^{\frac{1}{2}}\left(e^{c \int_{t}^{+\infty} s \log ^{2}(1+s)|\zeta(s)| d s}-1\right), \\
\left|E^{\prime}(t)\right| \leqslant t^{-\frac{1}{2}}\left(e^{c \int_{t}^{+\infty} s \log ^{2}(1+s)|\zeta(s)| d s}-1\right) .
\end{gathered}
$$

The proof is similar to the one of Theorem 1.8. 


\subsection{Estimates for $\Phi_{\lambda}$}

The next Theorem is essentially due to Bloom and $\mathrm{Xu}$ (see [2, Lemma 3.4]). We include a proof for reader's convenience.

Theorem 1.17. Assume that $(\mathrm{H} 1)$ to $(\mathrm{H} 4)$ hold and let $G \in L^{1}(1,+\infty)$. Then, for every $\lambda \in \mathbb{C}^{*}$, the differential equation

$$
\mathcal{L} u=\left(\lambda^{2}+\rho^{2}\right) u
$$

has a unique solution $\Phi_{\lambda}(t)$ over $(0,+\infty)$ which is twice continuously differentiable and satisfies

$$
A^{1 / 2}(t) \Phi_{\lambda}(t)=e^{i \lambda t}+e^{i \lambda t} \mathcal{R}(\lambda, t),
$$

with

$$
\begin{aligned}
& \mathcal{R}(\lambda, t) \rightarrow 0 \text { as } t \rightarrow \infty, \\
& \frac{\partial \mathcal{R}}{\partial t}(\lambda, t) \rightarrow 0 \text { as } t \rightarrow \infty .
\end{aligned}
$$

Moreover, for $\operatorname{Im}(\lambda) \geqslant 0, \lambda \neq 0$, and $t>0$

$$
\begin{aligned}
|\mathcal{R}(\lambda, t)| & \leqslant e^{\frac{1}{\lambda \mid} \int_{t}^{\infty}|q(v)| d v}-1 \\
\left|\frac{\partial}{\partial t} \mathcal{R}(\lambda, t)\right| & \leqslant|\lambda|\left(e^{\frac{1}{|\lambda|} \int_{t}^{\infty}|q(v)| d v}-1\right) .
\end{aligned}
$$

Proof. Let $\lambda \neq 0$. After the Liouville transformation $\sqrt{A(t)} u(t)=v(t)$, the above equation becomes equation (0.5)

$$
v^{\prime \prime}+\left(\lambda^{2}-\frac{\alpha^{2}-\frac{1}{4}}{t^{2}}\right) v-G(t) v=0 .
$$

Replacing $v(t)$ with $e^{i \lambda t}+e^{i \lambda t} \mathcal{R}(\lambda, t)$, we obtain

$$
\frac{d^{2}}{d t^{2}} \mathcal{R}(\lambda, t)+2 i \lambda \frac{d}{d t} \mathcal{R}(\lambda, t)=q(t) \mathcal{R}(\lambda, t)+q(t) .
$$

The homogeneous equation

$$
\frac{d^{2}}{d t^{2}} \mathcal{R}(\lambda, t)+2 i \lambda \frac{d}{d t} \mathcal{R}(\lambda, t)=0
$$


has the linearly independent solutions 1 and $e^{-2 i \lambda t}$ with Wronskian

$$
\mathcal{W}\left(1, e^{-2 i \lambda t}\right)=-2 i \lambda e^{-2 i \lambda t}
$$

Assume now $\operatorname{Im}(\lambda) \geqslant 0$. In view of Lemma A.1, a solution of the integral equation

$$
\mathcal{R}(\lambda, t)=\int_{t}^{\infty} \frac{e^{-2 i \lambda(t-v)}-1}{2 i \lambda}\{q(v) \mathcal{R}(\lambda, v)+q(v)\} d v
$$

also satisfies equation (1.17). We now apply Theorem (A.2) with $k(t, v)=$ $\frac{e^{-2 i \lambda(t-v)}-1}{2 i \lambda}, \phi(v)=\psi_{0}(v)=q(v)$ and $J(v) \equiv 1$. Since $\operatorname{Im}(\lambda) \geqslant 0$ a standard computation shows that we can set $P_{0}(t)=\frac{1}{|\lambda|}, P_{1}(t)=1, Q(v)=1$. Therefore there is a unique solution $\mathcal{R}(\lambda, t)$ of the integral equation which is twice continuously differentiable in $(0,+\infty)$ and satisfies

$$
\begin{gathered}
\mathcal{R}(\lambda, t) \rightarrow 0 \text { as } t \rightarrow \infty, \\
\frac{\partial}{\partial t} \mathcal{R}(\lambda, t) \rightarrow 0 \text { as } t \rightarrow \infty
\end{gathered}
$$

Furthermore

$$
|\mathcal{R}(\lambda, t)| \leqslant e^{\frac{1}{|\lambda|} \int_{t}^{\infty}|q(v)| d v}-1,
$$

and

$$
\left|\frac{\partial}{\partial t} \mathcal{R}(\lambda, t)\right| \leqslant|\lambda|\left(e^{\frac{1}{\lambda \mid} \int_{t}^{\infty}|q(v)| d v}-1\right) .
$$

When $\operatorname{Im}(\lambda)<0$, we consider the integral equation

$$
\mathcal{R}(\lambda, t)=-\int_{b}^{t} \frac{e^{-2 i \lambda(t-v)}-1}{2 i \lambda}\{q(v) \mathcal{R}(\lambda, v)+q(v)\} d v
$$

instead. Its solution also satisfies equation (1.17). Applying Theorem A.2 as before we obtain a unique solution $\widetilde{\mathcal{R}}(\lambda, t)$ continuously differentiable in $(b,+\infty)$ and satisfying

$$
\begin{aligned}
& \widetilde{\mathcal{R}}(\lambda, t) \rightarrow 0 \text { as } t \rightarrow b, \\
& \frac{\partial}{\partial t} \widetilde{\mathcal{R}}(\lambda, t) \rightarrow 0 \text { as } t \rightarrow b .
\end{aligned}
$$

Furthermore

$$
|\widetilde{\mathcal{R}}(\lambda, t)| \leqslant e^{\frac{1}{|\lambda|} \int_{b}^{t}|q(v)| d v}-1,
$$

and

$$
\left|\frac{\partial}{\partial t} \widetilde{\mathcal{R}}(\lambda, t)\right| \leqslant|\lambda|\left(e^{\frac{1}{|\lambda|} \int_{b}^{t}|q(v)| d v}-1\right) .
$$


Observe that $\widetilde{\mathcal{R}}(\lambda, \infty)=\lim _{t \rightarrow \infty} \widetilde{\mathcal{R}}(\lambda, t)$ exists and is finite. Indeed,

$$
\begin{aligned}
\widetilde{\mathcal{R}}(\lambda, \infty)= & \lim _{t \rightarrow \infty} \int_{b}^{t} \frac{1-e^{-2 i \lambda(t-v)}}{2 i \lambda}[q(v) \widetilde{\mathcal{R}}(\lambda, v)+q(v)] d v \\
= & \frac{1}{2 i \lambda} \lim _{t \rightarrow \infty}\left\{\int_{b}^{t}[q(v) \widetilde{\mathcal{R}}(\lambda, v)+q(v)] d v\right. \\
& -\int_{b}^{t / 2} e^{-2 i \lambda(t-v)}[q(v) \widetilde{\mathcal{R}}(\lambda, v)+q(v)] d v \\
& \left.-\int_{t / 2}^{t} e^{-2 i \lambda(t-v)}[q(v) \widetilde{\mathcal{R}}(\lambda, v)+q(v)] d v\right\},
\end{aligned}
$$

and this limit exists because $\widetilde{\mathcal{R}}(\lambda, v)$ is bounded, $q(v)$ is integrable near infinity, and

$$
\begin{aligned}
& \left|\int_{b}^{t / 2} e^{-2 i \lambda(t-v)}\{q(v) \widetilde{\mathcal{R}}(\lambda, v)+q(v)\} d v\right| \leqslant C e^{2 \operatorname{Im} \lambda t / 2} \int_{b}^{t / 2}|q(v)| d v \rightarrow 0 \\
& \left|\int_{t / 2}^{t} e^{-2 i \lambda(t-v)}\{q(v) \widetilde{\mathcal{R}}(\lambda, v)+q(v)\} d v\right| \leqslant C \int_{t / 2}^{t}|q(v)| d v \rightarrow 0
\end{aligned}
$$

as $t \rightarrow \infty$. Thus $\widetilde{\mathcal{R}}(\lambda, \infty)=\frac{1}{2 i \lambda} \int_{b}^{\infty}\{q(v) \widetilde{\mathcal{R}}(\lambda, v)+q(v)\} d v$. It is easy to show that the function

$$
\mathcal{R}(\lambda, t)=\frac{\widetilde{\mathcal{R}}(\lambda, t)-\widetilde{\mathcal{R}}(\lambda, \infty)}{1+\widetilde{\mathcal{R}}(\lambda, \infty)}
$$

(the denominator is different from 0 as long as $b$ is big enough) is a solution of (1.17) too. Furthermore,

$$
\mathcal{R}(\lambda, t) \rightarrow 0 \text { as } t \rightarrow \infty
$$

and

$$
\begin{aligned}
\frac{\partial \mathcal{R}}{\partial t}(\lambda, t) & =\frac{1}{1+\widetilde{\mathcal{R}}(\lambda, \infty)} \frac{\partial \widetilde{\mathcal{R}}}{\partial t}(\lambda, t)= \\
& =\frac{1}{1+\widetilde{\mathcal{R}}(\lambda, \infty)}\left\{\int_{b}^{t} e^{2 i \lambda(v-t)}\{q(v) \widetilde{\mathcal{R}}(\lambda, v)+q(v)\} d v\right\} \rightarrow 0
\end{aligned}
$$

as $t \rightarrow \infty$.

We have shown that the equation $\mathcal{L} u=\left(\lambda^{2}+\rho^{2}\right) u$ has a solution $\Phi_{\lambda}$ that satisfies (1.14), (1.15) and (1.16). Observe now that $\Phi_{-\lambda}$ is an independent solution of the same equation (see the next corollary for an explicit computation of the Wronskian). It follows that any solution can be written as $c_{1} \Phi_{\lambda}+c_{2} \Phi_{-\lambda}$. This immediately implies the uniqueness of $\Phi_{\lambda}$. 
Corollary 1.18. Assume (H1) to (H4) be satisfied and let $G \in L^{1}(1,+\infty)$. If $\lambda \in \mathbb{C}^{*}$ then the functions $\Phi_{\lambda}(t)$ and $\Phi_{-\lambda}(t)$ are linearly independent solutions of the equation

$$
\mathcal{L} u=\left(\lambda^{2}+\rho^{2}\right) u
$$

and their Wronskian is given by

$$
\mathcal{W}\left(\Phi_{\lambda}, \Phi_{-\lambda}\right)(t)=-2 i \lambda A(t)^{-1}
$$

Proof. It is enough to show that $\mathcal{W}\left(A^{1 / 2} \Phi_{\lambda}, A^{1 / 2} \Phi_{-\lambda}\right)=-2 i \lambda$. First observe that $\mathcal{W}\left(A^{1 / 2} \Phi_{\lambda}, A^{1 / 2} \Phi_{-\lambda}\right)$ is constant in $t$, since the coefficient of $u^{\prime}$ in equation (0.5) vanishes. Thus,

$$
\begin{aligned}
& \mathcal{W}\left(A^{1 / 2} \Phi_{\lambda}, A^{1 / 2} \Phi_{-\lambda}\right)=\lim _{t \rightarrow \infty} \mathcal{W}\left(A^{1 / 2}(t) \Phi_{\lambda}(t), A^{1 / 2}(t) \Phi_{-\lambda}(t)\right) \\
& =\lim _{t \rightarrow \infty} \mathcal{W}\left(e^{i \lambda t}(1+\mathcal{R}(\lambda, t)), e^{-i \lambda t}(1+\mathcal{R}(-\lambda, t))\right) \\
& =\lim _{t \rightarrow \infty}-2 i \lambda(1+\mathcal{R}(\lambda, t))(1+\mathcal{R}(-\lambda, t))+\mathcal{W}(1+\mathcal{R}(\lambda, t), 1+\mathcal{R}(-\lambda, t)) \\
& =-2 i \lambda .
\end{aligned}
$$

Following, and partially modifying [2], we define

$$
\begin{gathered}
\tilde{\Phi}_{-\lambda}(t)=\left\{\begin{array}{lr}
\lambda^{a-1 / 2} \Phi_{-\lambda}(t) & \text { when } a>0, \\
\lambda^{-1 / 2}\left(\log \frac{2}{\lambda}\right)^{-1} \Phi_{-\lambda}(t) & \text { when } a=0,
\end{array}\right. \\
C_{1}(a)= \begin{cases}\frac{2^{a-\frac{1}{2}} \Gamma(a)}{\sqrt{\pi}} e^{-i(2 a-1) \frac{\pi}{4}} & \text { when } a>0, \\
\sqrt{\frac{2}{\pi} e^{i \frac{\pi}{4}}} & \text { when } a=0,\end{cases}
\end{gathered}
$$

and

$$
C_{2}(a)= \begin{cases}\frac{e^{i \frac{(2 a-3) \pi}{4}} \Gamma(1-a)}{2^{a+\frac{1}{2}} a \sqrt{\pi}} & \text { when } 0<a<\frac{1}{2}, \\ \sqrt{\frac{\pi}{2}} e^{-i \frac{\pi}{4}}\left(1-\frac{2 \gamma}{\pi} i\right) & \text { when } a=0\end{cases}
$$

where $\gamma=0.57721566 \ldots$ denotes the Euler constant. Also let $W_{1}$ as in Theorem 1.8 and $W_{2}$ as in Theorem 1.16.

The next three theorems describe the analyticity properties of $\lambda \mapsto \widetilde{\Phi}_{-\lambda}(t)$ and its behavior as $\lambda \rightarrow 0$. The first one is contained in [2]. 
Theorem 1.19. Assume (H1) to (H5) hold. Then for each $t>1$ we can write

$$
\sqrt{A(t)} \widetilde{\Phi}_{-\lambda}(t)=C_{1}(a) W_{1}(t)+\mathcal{Z}_{\lambda}(t)
$$

with $\lambda \mapsto \mathcal{Z}_{\lambda}(t)$ and $\lambda \mapsto \frac{d}{d t} \mathcal{Z}_{\lambda}(t)$ analytic in $\{\operatorname{Im}(\lambda)<0\}$ and continuous in $\{\operatorname{Im}(\lambda) \leqslant 0\}$. Moreover

$$
\lim _{\substack{\lambda \rightarrow 0 \\ \operatorname{Im} \lambda \leqslant 0}} \mathcal{Z}_{\lambda}(t)=0
$$

and

$$
\lim _{\substack{\lambda \rightarrow 0 \\ \operatorname{Im} \lambda \leqslant 0}} \frac{d}{d t} \mathcal{Z}_{\lambda}(t)=0 .
$$

In the case $A(t)$ recessive we will need more precise information on the behavior of $\mathcal{Z}_{\lambda}(t)$ as $\lambda \rightarrow 0$. This situation happens only if $-\frac{1}{2}<b \leqslant 0$. We address this case in the following two theorems. Recall that $a=|b|$.

Theorem 1.20. Assume (H1) to (H6) hold with $-\frac{1}{2}<b<0$. Then

$$
\sqrt{A(t)} \widetilde{\Phi}_{-\lambda}(t)=C_{1}(a) W_{1}(t)+C_{2}(a) \lambda^{2 a} W_{2}(t)+\lambda^{2 a} Z_{\lambda}(t)
$$

and for every $t>1$, the functions $\lambda \mapsto Z_{\lambda}(t)$ and $\lambda \mapsto \frac{d}{d t} Z_{\lambda}(t)$ are analytic in $\operatorname{Im} \lambda<0$ and continuous in $\operatorname{Im} \lambda \leqslant 0$. Moreover

$$
\lim _{\substack{\lambda \rightarrow 0 \\ \operatorname{Im} \lambda \leqslant 0}} Z_{\lambda}(t)=0
$$

and

$$
\lim _{\substack{\lambda \rightarrow 0 \\ \operatorname{Im} \lambda \leqslant 0}} \frac{d}{d t} Z_{\lambda}(t)=0 .
$$

Theorem 1.21. Assume (H1) to (H6) with $b=0$ and $A(t)$ recessive. Then

$$
\sqrt{A(t)} \widetilde{\Phi}_{-\lambda}(t)=C_{1}(0) W_{1}(t)+\frac{C_{2}(0) W_{1}(t)-C_{1}(0) W_{2}(t)}{\log \frac{2}{\lambda}}+\frac{Z_{\lambda}(t)}{\log \frac{2}{\lambda}}
$$

and for every $t>1$, the functions $\lambda \mapsto Z_{\lambda}(t)$ and $\lambda \mapsto \frac{d}{d t} Z_{\lambda}(t)$ are analytic in $\operatorname{Im} \lambda<0$ and continuous in $\operatorname{Im} \lambda \leqslant 0$. Moreover

$$
\lim _{\substack{\lambda \rightarrow 0 \\ \operatorname{Im} \lambda \leqslant 0}} Z_{\lambda}(t)=0
$$

and

$$
\lim _{\substack{\lambda \rightarrow 0 \\ \operatorname{Im} \lambda \leqslant 0}} \frac{d}{d t} Z_{\lambda}(t)=0 .
$$


Proof of Theorem 1.19. Observe that, by $(0.5), \sqrt{A(t)} \Phi_{-\lambda}(t)$ is a solution of

$$
v^{\prime \prime}(t)+\left(\lambda^{2}-\frac{a^{2}-\frac{1}{4}}{t^{2}}\right) v(t)=\zeta(t) v(t) .
$$

The associated homogeneous equation

$$
v^{\prime \prime}(t)+\left(\lambda^{2}-\frac{a^{2}-\frac{1}{4}}{t^{2}}\right) v(t)=0
$$

has the two linearly independent solutions $\mathcal{H}_{a}^{(2)}(\lambda t)$ and $\mathcal{J}_{a}(\lambda t)$ with Wronskian

$$
\mathcal{W}\left(\mathcal{H}_{a}^{(2)}(\lambda t), \mathcal{J}_{a}(\lambda t)\right)=\frac{2 i \lambda}{\pi}
$$

By Lemma A.1, a solution of the integral equation

$$
v(t)=C(a) \mathcal{H}_{a}^{(2)}(\lambda t)+\int_{t}^{\infty} k_{1}(t, s, \lambda) e^{i \lambda(s-t)} v(s) d s
$$

where

$$
k_{1}(t, s, \lambda)=\frac{\pi}{2 i \lambda}\left(\mathcal{H}_{a}^{(2)}(\lambda t) \mathcal{J}_{a}(\lambda s)-\mathcal{J}_{a}(\lambda t) \mathcal{H}_{a}^{(2)}(\lambda s)\right) \zeta(s) e^{i \lambda(t-s)}
$$

also solves (1.22).

We will show later that for a suitable choice of $C(a), \sqrt{A(t)} \Phi_{-\lambda}(t)$ is a solution of (1.23).

Assume now $a>0$ and let $\widetilde{v}_{\lambda}(t)=\lambda^{a-\frac{1}{2}} v(t) e^{i \lambda t}$. With this notation the above equation reads

$$
\tilde{v}_{\lambda}(t)=C(a) \lambda^{a-\frac{1}{2}} \mathcal{H}_{a}^{(2)}(\lambda t) e^{i \lambda t}+\int_{t}^{\infty} k_{1}(t, s, \lambda) \widetilde{v}_{\lambda}(s) d s .
$$

When $t \leqslant s$ and $\operatorname{Im} \lambda \leqslant 0$ the expansions for Bessel and Hankel functions give

$$
\begin{gathered}
\left|k_{1}(t, s, \lambda)\right| \leqslant C \frac{\langle\lambda t\rangle^{-a+1 / 2}}{|\lambda|}\langle\lambda s\rangle^{a+1 / 2}|\zeta(s)| \\
\left|\frac{\partial k_{1}}{\partial t}(t, s, \lambda)\right| \leqslant C\langle\lambda t\rangle^{-a-1 / 2}\langle\lambda s\rangle^{a+1 / 2}|\zeta(s)| .
\end{gathered}
$$

Theorem A.3 applied to the integral equation

$$
\tilde{v}_{\lambda}(t)=C(a) \lambda^{a-\frac{1}{2}} \mathcal{H}_{a}^{(2)}(\lambda t) e^{i \lambda t}+\int_{t}^{\infty} \frac{k_{1}(t, s, \lambda)}{s \zeta(s)} s \zeta(s) \widetilde{v}_{\lambda}(s) d s
$$


with

$$
\begin{aligned}
\phi(s) & =s \zeta(s) \\
J(s) & =C(a) \lambda^{a-\frac{1}{2}} \mathcal{H}_{a}^{(2)}(\lambda t) e^{i \lambda t} \\
Q(s) & =C s^{-1}\langle\lambda s\rangle^{a+1 / 2} \\
P_{0}(t) & =|\lambda|^{-1}\langle\lambda t\rangle^{-a+1 / 2} \\
P_{1}(t) & =\langle\lambda t\rangle^{-a-1 / 2} \\
\kappa & =C|\lambda|^{a+\frac{1}{2}} \\
\kappa_{0} & =C
\end{aligned}
$$

shows that there is one solution $\widetilde{v}_{\lambda}(t)$ such that

$$
\begin{aligned}
\widetilde{v}_{\lambda}(t)-C(a) \lambda^{a-\frac{1}{2}} \mathcal{H}_{a}^{(2)}(\lambda t) e^{i \lambda t} & \rightarrow 0, \\
\frac{\partial}{\partial t}\left(\widetilde{v}_{\lambda}(t)-C(a) \lambda^{a-\frac{1}{2}} \mathcal{H}_{a}^{(2)}(\lambda t) e^{i \lambda t}\right) & \rightarrow 0
\end{aligned}
$$

as $t \rightarrow \infty$. Let now

$$
C(a)=\sqrt{\frac{\pi}{2}} e^{-i \frac{(2 a+1) \pi}{4}}
$$

so that $C(a) \mathcal{H}_{a}^{(2)}(\lambda t) e^{i \lambda t} \sim 1$ as $t \rightarrow+\infty$. Theorem (1.17) and the decay properties of $\widetilde{v}_{\lambda}(t)$ give

$$
\widetilde{v}_{\lambda}(t)=A^{1 / 2}(t) \widetilde{\Phi}_{-\lambda}(t) e^{i \lambda t}
$$

Theorem A. 3 also gives

$$
\begin{aligned}
& \left|\widetilde{v}_{\lambda}(t)-C(a) \lambda^{a-\frac{1}{2}} \mathcal{H}_{a}^{(2)}(\lambda t) e^{i \lambda t}\right| \\
& \leqslant C\left(\frac{t}{1+|\lambda| t}\right)^{-a+1 / 2}\left(e^{C \int_{t}^{\infty}|\zeta(s)||s| d s}-1\right)
\end{aligned}
$$

and

$$
\begin{aligned}
& \left|\frac{\partial}{\partial t}\left(\widetilde{v}_{\lambda}(t)-C(a) \lambda^{a-\frac{1}{2}} \mathcal{H}_{a}^{(2)}(\lambda t) e^{i \lambda t}\right)\right| \\
& \leqslant C\left(\frac{t}{1+|\lambda| t}\right)^{-a-1 / 2}\left(e^{C \int_{t}^{\infty}|\zeta(s)||s| d s}-1\right) .
\end{aligned}
$$

More precisely

$$
\widetilde{v}_{\lambda}=\sum_{j=0}^{\infty}\left(v_{j+1}(t, \lambda)-v_{j}(t, \lambda)\right)
$$


where

$$
v_{j+1}(t, \lambda)=C(a) \lambda^{a-\frac{1}{2}} \mathcal{H}_{a}^{(2)}(\lambda t) e^{i \lambda t}+\int_{t}^{\infty} \frac{k_{1}(t, s, \lambda)}{s \zeta(s)} s \zeta(s) v_{j}(s, \lambda) d s
$$

and $v_{0}(t, \lambda) \equiv 0$. Then for $\operatorname{Im} \lambda \leqslant 0$,

$$
\left|v_{j+1}(t, \lambda)-v_{j}(t, \lambda)\right| \leqslant C\left(\frac{t}{1+|\lambda| t}\right)^{-a+1 / 2} \frac{\left(C \int_{t}^{\infty} s|\zeta(s)| d s\right)^{j+1}}{j !}
$$

and

$$
\left|\frac{\partial v_{j+1}}{\partial t}(t, \lambda)-\frac{\partial v_{j}}{\partial t}(t, \lambda)\right| \leqslant C\left(\frac{t}{1+|\lambda t|}\right)^{-a-1 / 2} \frac{\left(C \int_{t}^{\infty}|\zeta(s)| s d s\right)^{j+1}}{j !} .
$$

This shows that $\widetilde{v}_{\lambda}(t)$ and $\widetilde{v}_{\lambda}^{\prime}(t)$ are analytic in $\{\operatorname{Im} \lambda<0\}$ and continuous in $\{\operatorname{Im} \lambda \leqslant 0\}$.

In order to show (1.18) and (1.19) we observe that the estimates for $\widetilde{v}_{\lambda}$ and $\widetilde{v}_{\lambda}^{\prime}$ allow to apply the dominated convergence theorem in 1.26. Since

$$
\lim _{\lambda \rightarrow 0} \lambda^{a-\frac{1}{2}} \mathcal{H}_{a}^{(2)}(\lambda t)=\frac{i 2^{a} \Gamma(a)}{\pi} t^{\frac{1}{2}-a}
$$

letting $\lambda \rightarrow 0$ gives

$$
\widetilde{v}_{0}(t)=C_{1}(a) t^{\frac{1}{2}-a}+\int_{t}^{\infty} k(t, s) \widetilde{v}_{0}(s) d s
$$

where $k(t, s)$ is defined by (1.8) and $C_{1}(a)=C(a) \frac{i 2^{a} \Gamma(a)}{\pi}$.

Also by (1.28) and (1.29) we have

$$
\begin{aligned}
& \left|\widetilde{v}_{0}(t)-C_{1}(a) t^{\frac{1}{2}-a}\right| \leqslant C t^{-a+1 / 2}\left(e^{C \int_{t}^{\infty}|\zeta(s)||s| d s}-1\right), \\
& \left|\widetilde{v}_{0}^{\prime}(t)-\left(\frac{1}{2}-a\right) C_{1}(a) t^{-a-\frac{1}{2}}\right| \leqslant C t^{-a-1 / 2}\left(e^{C \int_{t}^{\infty}|\zeta(s)||s| d s}-1\right) .
\end{aligned}
$$

By Theorem 1.8 we see that $\frac{\widetilde{v}_{0}(t)}{C_{1}(a)}$ satisfies the same integral equation as $W_{1}(t)$. The above estimates guarantee the uniqueness of the solution of the integral equation and we finally obtain $\widetilde{v}_{0}(t)=C_{1}(a) W_{1}(t)$ and therefore (1.18). Differentiating (1.26) and letting $\lambda \rightarrow 0$ gives

$$
\begin{aligned}
\lim _{\substack{\lambda \rightarrow 0 \\
\operatorname{Im} \lambda \leqslant 0}} \widetilde{v}_{\lambda}^{\prime}(t) & =\left(\frac{1}{2}-a\right) C_{1}(a) t^{-a-\frac{1}{2}}+\int_{t}^{\infty} \frac{\partial k}{\partial t}(t, s) C_{1}(a) W_{1}(s) d s \\
& =C_{1}(a) W_{1}^{\prime}(t) .
\end{aligned}
$$


Assume now $a=0$, and let $\widetilde{v}_{\lambda}(t)=\lambda^{-\frac{1}{2}}\left(\log \frac{2}{\lambda}\right)^{-1} v(t) e^{i \lambda t}$. With this notation equation (1.23) reads

$$
\widetilde{v}_{\lambda}(t)=C(0) \lambda^{-\frac{1}{2}}\left(\log \frac{2}{\lambda}\right)^{-1} \mathcal{H}_{0}^{(2)}(\lambda t) e^{i \lambda t}+\int_{t}^{\infty} k_{1}(t, s, \lambda) \widetilde{v}_{\lambda}(s) d s
$$

When $|\lambda| s \geqslant 1$ and $\operatorname{Im} \lambda \leqslant 0$, the standard expansions for Bessel and Hankel functions give

$$
\begin{gathered}
\left|k_{1}(t, s, \lambda)\right| \leqslant C|\lambda|^{-1}\langle\lambda t\rangle^{1 / 2} \log \left(\frac{2}{\langle\lambda t\rangle}\right)\langle\lambda s\rangle^{1 / 2}|\zeta(s)| \\
\left|\frac{\partial k_{1}}{\partial t}(t, s, \lambda)\right| \leqslant C\langle\lambda t\rangle^{-1 / 2} \log \left(\frac{2}{\langle\lambda t\rangle}\right)\langle\lambda s\rangle^{1 / 2}|\zeta(s)| .
\end{gathered}
$$

For the remaining case, $|\lambda| t \leqslant|\lambda| s \leqslant 1$, observe that $\mathcal{H}_{0}^{(2)}(\lambda t) \mathcal{J}_{0}(\lambda s)-\mathcal{J}_{0}(\lambda t) \mathcal{H}_{0}^{(2)}(\lambda s)=-i \lambda \sqrt{t s}\left(Y_{0}(\lambda t) J_{0}(\lambda s)-Y_{0}(\lambda s) J_{0}(\lambda t)\right)$ and

$$
Y_{0}(z)=\frac{2}{\pi} J_{0}(z) \log \frac{z}{2}-\frac{2}{\pi} F(z)
$$

where

$$
F(z)=\sum_{k=0}^{+\infty} \frac{(-1)^{k}\left(\frac{z}{2}\right)^{2 k}}{(k !)^{2}}\left(-\gamma+\sum_{j=1}^{k} \frac{1}{j}\right)
$$

and $\gamma=0,577 \ldots$ is Euler's constant (see [10, Chapter 5]). Thus

$$
\begin{aligned}
\mathcal{H}_{0}^{(2)}(\lambda t) \mathcal{J}_{0}(\lambda s)-\mathcal{J}_{0}(\lambda t) \mathcal{H}_{0}^{(2)}(\lambda s) \\
=-i \lambda \sqrt{t s}\left[\left(\frac{2}{\pi} J_{0}(\lambda t) \log \frac{\lambda t}{2}-\frac{2}{\pi} F(\lambda t)\right) J_{0}(\lambda s)\right. \\
\left.\quad-\left(\frac{2}{\pi} J_{0}(\lambda s) \log \frac{\lambda s}{2}-\frac{2}{\pi} F(\lambda s)\right) J_{0}(\lambda t)\right] \\
=-\frac{2}{\pi} i \lambda \sqrt{t s}\left(J_{0}(\lambda t) J_{0}(\lambda s) \log \frac{t}{s}-F(\lambda t) J_{0}(\lambda s)+F(\lambda s) J_{0}(\lambda t)\right) .
\end{aligned}
$$


It is easy to see that, for $|\lambda| t \leqslant 1,|\sqrt{\lambda t} F(\lambda t)| \leqslant C \sqrt{\lambda t}$ and $\left|\frac{\partial}{\partial t}(\sqrt{\lambda t} F(\lambda t))\right| \leqslant$ $C|\lambda|(\sqrt{|\lambda| t})^{-1}$. Therefore, for $|\lambda| t \leqslant|\lambda| s \leqslant 1$, we trivially have

$$
\begin{aligned}
& \left|\mathcal{H}_{0}^{(2)}(\lambda t) \mathcal{J}_{0}(\lambda s)-\mathcal{J}_{0}(\lambda t) \mathcal{H}_{0}^{(2)}(\lambda s)\right| \\
& \leqslant C|\lambda| \sqrt{t s} \log \frac{2 s}{t} \approx\langle\lambda t\rangle^{\frac{1}{2}}\langle\lambda s\rangle^{\frac{1}{2}} \log \frac{2 s}{t} \\
& \left|\frac{\partial}{\partial t}\left[\mathcal{H}_{0}^{(2)}(\lambda t) \mathcal{J}_{0}(\lambda s)-\mathcal{J}_{0}(\lambda t) \mathcal{H}_{0}^{(2)}(\lambda s)\right]\right| \\
& \leqslant C|\lambda| \sqrt{\frac{s}{t}} \log \frac{2 s}{t} \approx|\lambda|\langle\lambda t\rangle^{-\frac{1}{2}}\langle\lambda s\rangle^{\frac{1}{2}} \log \frac{2 s}{t} .
\end{aligned}
$$

In the overall we have, for $t \leqslant s$ and $\operatorname{Im} \lambda \leqslant 0$,

$$
\left|k_{1}(t, s, \lambda)\right| \leqslant C|\lambda|^{-1}\langle\lambda t\rangle^{1 / 2}\langle\lambda s\rangle^{1 / 2} \log \frac{2\langle\lambda s\rangle}{\langle\lambda t\rangle}|\zeta(s)|
$$

and

$$
\left|\frac{\partial k_{1}}{\partial t}(t, s, \lambda)\right| \leqslant C\langle\lambda t\rangle^{-1 / 2}\langle\lambda s\rangle^{1 / 2} \log \frac{2\langle\lambda s\rangle}{\langle\lambda t\rangle}|\zeta(s)| .
$$

Since

$$
\log \frac{2\langle\lambda s\rangle}{\langle\lambda t\rangle} \leqslant c \log \frac{2}{\langle\lambda t\rangle}\left\langle\log (2 s)\left(\log \frac{2}{|\lambda|}\right)^{-1}\right\rangle
$$

we can write

$$
\begin{gathered}
\left|k_{1}(t, s, \lambda)\right| \leqslant c|\lambda|^{-1}\langle\lambda t\rangle^{1 / 2} \log \frac{2}{\langle\lambda t\rangle}\langle\lambda s\rangle^{1 / 2}\left\langle\frac{\log (2 s)}{\log \frac{2}{|\lambda|}}\right\rangle|\zeta(s)|, \\
\left|\frac{\partial k_{1}}{\partial t}(t, s, \lambda)\right| \leqslant c\langle\lambda t\rangle^{-1 / 2} \log \frac{2}{\langle\lambda t\rangle}\langle\lambda s\rangle^{1 / 2}\left\langle\frac{\log (2 s)}{\log \frac{2}{|\lambda|}}\right\rangle|\zeta(s)| .
\end{gathered}
$$

The proof now follows applying Theorem A.3 to

$$
\begin{aligned}
\widetilde{v}(t)= & C(0) \mathcal{H}_{0}^{(2)}(\lambda t) \lambda^{-1 / 2}\left(\log \frac{2}{\lambda}\right)^{-1} e^{i \lambda t} \\
& +\int_{t}^{\infty} \frac{k_{1}(t, s, \lambda)}{s \log (2 s) \zeta(s)}(s \log (2 s) \zeta(s) \widetilde{v}(s)) d s .
\end{aligned}
$$

as in the case $a>0$.

In order to prove Theorem 1.20 we need the following technical lemma. 
Lemma 1.22. Assume (H1) to (H6) hold with $-\frac{1}{2}<b<0$, let

$$
C(a)=\sqrt{\frac{\pi}{2}} e^{-i \frac{(2 a+1) \pi}{4}}
$$

and let

$$
\begin{aligned}
\Theta_{\lambda}(t)= & C(a) \mathcal{H}_{a}^{(2)}(\lambda t) \lambda^{-a-\frac{1}{2}} e^{i \lambda t}-C_{1}(a) \lambda^{-2 a} t^{\frac{1}{2}-a}-C_{2}(a) t^{\frac{1}{2}+a} \\
& +\int_{t}^{+\infty} \frac{k_{1}(t, s, \lambda)-k(t, s)}{\lambda^{2 a}}\left(C_{1}(a) W_{1}(s)+C_{2}(a) \lambda^{2 a} W_{2}(s)\right) d s
\end{aligned}
$$

where $k_{1}$ is as in the proof of Theorem 1.19 and $k$ is as in Theorem 1.8. Then uniformly in $\operatorname{Im} \lambda \leqslant 0$,

$$
\left|\Theta_{\lambda}(t)\right| \leqslant c t^{\frac{1}{2}+a} .
$$

Also for every fixed $t \geqslant 1$ we have

$$
\lim _{\substack{\lambda \rightarrow 0 \\ \operatorname{Im} \lambda \leqslant 0}} \Theta_{\lambda}(t)=0
$$

and

$$
\lim _{\substack{\lambda \rightarrow 0 \\ \operatorname{Im} \lambda \leqslant 0}} \Theta_{\lambda}^{\prime}(t)=0 .
$$

Proof. Since $H_{a}^{(2)}(z)=\frac{e^{a \pi i} J_{a}(z)-J_{-a}(z)}{i \sin \pi a}$ (see [15, Chapter III, Section 3.61]), from the asymptotic expansions of $J_{a}$ we have

$$
\mathcal{H}_{a}^{(2)}(\lambda t)=\frac{e^{i \pi a} \frac{(\lambda t)^{a+\frac{1}{2}}}{2^{a} \Gamma(a+1)}-\frac{(\lambda t)^{-a+\frac{1}{2}}}{2^{-a} \Gamma(-a+1)}}{i \sin \pi a}+O\left((\lambda t)^{\frac{5}{2}-a}\right)
$$

when $|\lambda| t \leqslant 1$. A few computations give

$$
\begin{aligned}
& C(a) \lambda^{-a-\frac{1}{2}} \mathcal{H}_{a}^{(2)}(\lambda t) e^{i \lambda t}-C_{1}(a) \lambda^{-2 a} t^{\frac{1}{2}-a}-C_{2}(a) t^{\frac{1}{2}+a} \\
& =O\left(\lambda^{1-2 a} t^{\frac{3}{2}-a}\right)=O\left(t^{\frac{1}{2}+a}\right) .
\end{aligned}
$$

Observe that since $a<\frac{1}{2}$ the estimate $O\left(\lambda^{1-2 a} t^{\frac{3}{2}-a}\right)$ implies that the above expression goes to zero as $\lambda \rightarrow 0$.

In the case $|\lambda t| \geqslant 1$, the standard estimates on the Hankel function give

$$
\begin{aligned}
& \left|C(a) \lambda^{-a-\frac{1}{2}} \mathcal{H}_{a}^{(2)}(\lambda t) e^{i \lambda t}-C_{1}(a) \lambda^{-2 a} t^{\frac{1}{2}-a}-C_{2}(a) t^{\frac{1}{2}+a}\right| \\
& \leqslant\left|C(a) \lambda^{-a-\frac{1}{2}} \mathcal{H}_{a}^{(2)}(\lambda t) e^{i \lambda t}\right|+\left|C_{1}(a) \lambda^{-2 a} t^{\frac{1}{2}-a}\right|+\left|C_{2}(a) t^{\frac{1}{2}+a}\right| \\
& \leqslant c t^{\frac{1}{2}+a} .
\end{aligned}
$$


We now estimate the integral part in the definition of $\Theta_{\lambda}(t)$. When $|\lambda t|<1$ we estimate separately the integral on $(t, 1 /|\lambda|)$ and the integral on $(1 /|\lambda|,+\infty)$.

The standard expansions for $J_{a}$ and $J_{-a}$ along with the identity $H_{a}^{(2)}(z)=$ $\frac{e^{a \pi i} J_{a}(z)-J_{-a}(z)}{i \sin (a \pi)}$, give for $|\lambda t| \leqslant|\lambda s| \leqslant 1$

$$
\left|\frac{k_{1}(t, s, \lambda)-k(t, s)}{\lambda^{2 a}}\right| \leqslant c t^{-a+\frac{1}{2}} s^{a+\frac{5}{2}}|\lambda|^{2-2 a}|\zeta(s)|
$$

so that

$$
\begin{aligned}
& \int_{t}^{1 /|\lambda|}\left|\frac{k_{1}(t, s, \lambda)-k(t, s)}{\lambda^{2 a}}\right|\left|C_{1}(a) W_{1}(s)+C_{2}(a) \lambda^{2 a} W_{2}(s)\right| d s \\
& \leqslant c t^{-a+\frac{1}{2}} \int_{t}^{1 /|\lambda|} s^{2-2 a}|\lambda|^{2-2 a} s^{1+2 a}|\zeta(s)| d s \leqslant c t^{-a+\frac{1}{2}} \int_{t}^{+\infty} s^{1+2 a}|\zeta(s)| d s .
\end{aligned}
$$

Since $a<\frac{1}{2}$ we have

$$
\frac{k_{1}(t, s, \lambda)-k(t, s)}{\lambda^{2 a}} \rightarrow 0
$$

as $\lambda \rightarrow 0$ in $\operatorname{Im} \lambda \leqslant 0$, and the dominated convergence theorem shows that

$$
\int_{t}^{1 /|\lambda|} \frac{k_{1}(t, s, \lambda)-k(t, s)}{\lambda^{2 a}}\left[C_{1}(a) W_{1}(s)+C_{2}(a) \lambda^{2 a} W_{2}(s)\right] d s \rightarrow 0 .
$$

When $|\lambda s| \geqslant 1$ by (1.25) we have

$$
\begin{aligned}
\left|k_{1}(t, s, \lambda)-k(t, s)\right| & \leqslant\left|k_{1}(t, s, \lambda)\right|+|k(t, s)| \\
& \leqslant c t^{-a+\frac{1}{2}}\left(|\lambda|^{-a-\frac{1}{2}}+s^{\frac{1}{2}+a}\right)|\zeta(s)| \\
& \leqslant c t^{-a+\frac{1}{2}} s^{\frac{1}{2}+a}|\zeta(s)|
\end{aligned}
$$

and therefore

$$
\begin{aligned}
& \int_{1 /|\lambda|}^{+\infty}\left|\frac{k_{1}(t, s, \lambda)-k(t, s)}{\lambda^{2 a}}\right|\left|C_{1}(a) W_{1}(s)+C_{2}(a) \lambda^{2 a} W_{2}(s)\right| d s \\
& \leqslant c t^{\frac{1}{2}-a} \int_{1 /|\lambda|}^{+\infty}|\zeta(s)| s^{1+2 a} d s
\end{aligned}
$$

that vanishes as $\lambda \rightarrow 0$ so that $\lim _{\operatorname{Im} \lambda \leqslant 0} \underset{\lambda \leqslant 0}{ } \Theta_{\lambda}(t)=0$. 
In the case $|\lambda t| \geqslant 1$ we have

$$
\begin{aligned}
& \int_{t}^{+\infty}\left|\frac{k_{1}(t, s, \lambda)-k(t, s)}{\lambda^{2 a}}\right|\left|C_{1}(a) W_{1}(s)+C_{2}(a) \lambda^{2 a} W_{2}(s)\right| d s \\
& \leqslant c \int_{t}^{+\infty}\left(|\lambda|^{-1}+t^{\frac{1}{2}-a} s^{\frac{1}{2}+a}\right)|\zeta(s)| s^{\frac{1}{2}+a} d s \\
& \leqslant c t^{\frac{1}{2}-a} \int_{t}^{+\infty}|\zeta(s)| s^{1+2 a} d s .
\end{aligned}
$$

In the overall

$$
\begin{aligned}
& \left|\int_{t}^{\infty} \frac{k_{1}(t, s, \lambda)-k(t, s)}{\lambda^{2 a}}\left(C_{1}(a) W_{1}(s)+C_{2}(a) \lambda^{2 a} W_{2}(s)\right) d s\right| \\
& \leqslant c t^{\frac{1}{2}-a} \int_{t}^{+\infty}|\zeta(s)| s^{1+2 a} d s \leqslant c t^{\frac{1}{2}+a} .
\end{aligned}
$$

The fact that $\lim _{\operatorname{Im} \lambda \leqslant 0} \Theta_{\lambda}^{\prime}(t)=0$ follows similarly.

Proof of Theorem 1.20. Since $\widetilde{\Phi}_{-\lambda}(t)$ is analytic in $\operatorname{Im} \lambda<0$ and continuous in $\operatorname{Im} \lambda \leqslant 0$ it suffices to consider the case $|\lambda|<1$. Let

$$
\widetilde{v}_{\lambda}(t)=\sqrt{A(t)} \widetilde{\Phi}_{-\lambda}(t) e^{i \lambda t}
$$

and

$$
\widetilde{Z}_{\lambda}(t)=\frac{\widetilde{v}_{\lambda}(t)-C_{1}(a) W_{1}(t)-C_{2}(a) \lambda^{2 a} W_{2}(t)}{\lambda^{2 a}} .
$$

We will show that the functions $\lambda \mapsto \widetilde{Z}_{\lambda}(t)$ and $\lambda \mapsto \widetilde{Z}_{\lambda}^{\prime}(t)$ are analytic in $\{\operatorname{Im} \lambda<0\}$ and continuous in $\{\operatorname{Im} \lambda \leqslant 0\}$, and that

$$
\lim _{\substack{\lambda \rightarrow 0 \\ \operatorname{Im} \lambda \leqslant 0}} \widetilde{Z}_{\lambda}(t)=0
$$

and

$$
\lim _{\substack{\lambda \rightarrow 0 \\ \operatorname{Im} \lambda \leqslant 0}} \widetilde{Z}_{\lambda}^{\prime}(t)=0
$$

The analogous results for the function $Z_{\lambda}$ follow easily.

By (1.24), (1.10) and (1.12)

$$
\widetilde{Z}_{\lambda}(t)=\Theta_{\lambda}(t)+\int_{t}^{+\infty} k_{1}(t, s, \lambda) \widetilde{Z}_{\lambda}(s) d s,
$$

where $\Theta_{\lambda}$ is as in Lemma 1.22. 
We now apply Theorem A.2 to the equation (1.32) that we rewrite in the following form

$$
\widetilde{Z}_{\lambda}(t)=\Theta_{\lambda}(t)+\int_{t}^{+\infty} \frac{k_{1}(t, s, \lambda)}{\zeta(s) s^{2 a+1}} \zeta(s) s^{2 a+1} \widetilde{Z}_{\lambda}(s) d s .
$$

For the kernel we have the estimates (see (1.25))

$$
\left|\frac{k_{1}(t, s, \lambda)}{\zeta(s) s^{2 a+1}}\right| \leqslant c \frac{\langle\lambda t\rangle^{-a+\frac{1}{2}}\langle\lambda s\rangle^{a+\frac{1}{2}}}{|\lambda| s^{2 a+1}}=P_{0}(t) Q(s)
$$

and

$$
\left|\frac{\frac{\partial k_{1}}{\partial t}(t, s, \lambda)}{\zeta(s) s^{2 a+1}}\right| \leqslant c \frac{\langle\lambda t\rangle^{-a-\frac{1}{2}}\langle\lambda s\rangle^{a+\frac{1}{2}}}{s^{2 a+1}}=P_{1}(t) Q(s)
$$

where $Q(s)=c \frac{\langle\lambda s\rangle^{a+\frac{1}{2}}}{s^{2 a+1}}, P_{0}(t)=\frac{\langle\lambda t\rangle^{-a+\frac{1}{2}}}{|\lambda|}$ and $P_{1}(t)=\langle\lambda t\rangle^{-a-\frac{1}{2}}$. A simple computation shows that $\kappa=c|\lambda|^{a+\frac{1}{2}}$ and $\kappa_{0}=c$. By Theorem A.3 equation (1.32) has a unique solution $u_{\lambda}(t)$ such that

$$
\lim _{t \rightarrow+\infty}\left[u_{\lambda}(t)-\Theta_{\lambda}(t)\right]=0
$$

and

$$
\lim _{t \rightarrow+\infty}\left[u_{\lambda}^{\prime}(t)-\Theta_{\lambda}^{\prime}(t)\right]=0 .
$$

Such a solution is given by

$$
u_{\lambda}(t)=\sum_{j=0}^{+\infty}\left[u_{j+1}(t, \lambda)-u_{j}(t, \lambda)\right]
$$

where

$$
u_{j+1}(t, \lambda)=\Theta_{\lambda}(t)+\int_{t}^{+\infty} k_{1}(t, s, \lambda) u_{j}(s, \lambda) d s
$$

and $u_{0} \equiv 0$. Moreover

$$
\left|u_{j+1}(t, \lambda)-u_{j}(t, \lambda)\right| \leqslant c \frac{t^{-a+\frac{1}{2}}}{(1+|\lambda| t)^{-a+\frac{1}{2}}} \frac{\left(c \int_{t}^{+\infty}|\zeta(s)| s^{2 a+1} d s\right)^{j+1}}{j !}
$$

and

$$
\left|\frac{\partial u_{j+1}}{\partial t}(t, \lambda)-\frac{\partial u_{j}}{\partial t}(t, \lambda)\right| \leqslant c \frac{t^{-a-\frac{1}{2}}}{(1+|\lambda t|)^{-a-\frac{1}{2}}} \frac{\left(c \int_{t}^{+\infty}|\zeta(s)| s^{2 a+1} d s\right)^{j+1}}{j !} .
$$


Therefore the above series and its derivative converge uniformly in $\lambda$ and we have the estimates

$$
\left|u_{\lambda}(t)-\Theta_{\lambda}(t)\right| \leqslant \frac{t^{-a+\frac{1}{2}}}{(1+|\lambda t|)^{-a+\frac{1}{2}}}\left(e^{c \int_{t}^{+\infty}|\zeta(s)| s^{1+2 a} d s}-1\right)
$$

and

$$
\left|u_{\lambda}^{\prime}(t)-\Theta_{\lambda}^{\prime}(t)\right| \leqslant \frac{t^{-a-\frac{1}{2}}}{(1+|\lambda t|)^{-a-\frac{1}{2}}}\left(e^{c \int_{t}^{+\infty}|\zeta(s)| s^{1+2 a} d s}-1\right) .
$$

By (1.32) we have

$$
\begin{aligned}
\widetilde{Z}_{\lambda}(t)-\Theta_{\lambda}(t) & =\int_{t}^{+\infty} k_{1}(t, s, \lambda) \widetilde{Z}_{\lambda}(s) d s \\
& =\frac{\int_{t}^{+\infty} k_{1}(t, s, \lambda)\left(\widetilde{v}_{\lambda}(s)-C_{1}(a) W_{1}(s)-C_{2}(a) \lambda^{2 a} W_{2}(s)\right) d s}{\lambda^{2 a}} .
\end{aligned}
$$

Using the estimates on $k_{1}, \Phi_{-\lambda}, W_{1}$ and $W_{2}$ we obtain that for large $t$

$$
\begin{aligned}
& \left|\tilde{Z}_{\lambda}(t)-\Theta_{\lambda}(t)\right| \\
& \leqslant \frac{1}{|\lambda|^{2 a}} \int_{t}^{+\infty}\left|k_{1}(t, s, \lambda)\left(\widetilde{v}_{\lambda}(s)-C_{1}(a) W_{1}(s)-C_{2}(a) \lambda^{2 a} W_{2}(s)\right)\right| d s \\
& \leqslant C \frac{1}{|\lambda|^{1+2 a}} \int_{t}^{+\infty} s^{2 a+1}|\zeta(s)| d s .
\end{aligned}
$$

It follows that

$$
\lim _{t \rightarrow+\infty}\left[\widetilde{Z}_{\lambda}(t)-\Theta_{\lambda}(t)\right]=0 .
$$

A similar estimate shows that

$$
\lim _{t \rightarrow+\infty}\left[\widetilde{Z}_{\lambda}^{\prime}(t)-\Theta_{\lambda}^{\prime}(t)\right]=0
$$

and by the uniqueness of the solution of (1.32) we have $u_{\lambda}(t)=\widetilde{Z}_{\lambda}(t)$. To show $\lim _{\operatorname{Im} \lambda \leqslant 0} \widetilde{Z}_{\lambda}(t)=0$ observe that

$$
\left|u_{j}(t, \lambda)\right| \leqslant c_{j} t^{\frac{1}{2}+a}
$$

and

$$
\lim _{\substack{\lambda \rightarrow 0 \\ \operatorname{Im} \lambda \leqslant 0}} u_{j}(t, \lambda)=0
$$

Indeed, since

$$
u_{1}(t, \lambda)=\Theta_{\lambda}(t)
$$


we have, by induction,

$$
\begin{aligned}
\left|u_{j+1}(t, \lambda)\right| & \leqslant O\left(t^{\frac{1}{2}+a}\right)+c_{j} \int_{t}^{+\infty}\left|k_{1}(t, s, \lambda)\right| s^{\frac{1}{2}+a} d s \\
& \leqslant O\left(t^{\frac{1}{2}+a}\right)+c_{j} t^{-a+\frac{1}{2}} \int_{t}^{+\infty}|\zeta(s)| s^{2 a+1} d s \\
& =O\left(t^{\frac{1}{2}+a}\right) .
\end{aligned}
$$

By the dominated convergence theorem we have

$$
\lim _{\substack{\lambda \rightarrow 0 \\ \operatorname{Im} \lambda \leqslant 0}} u_{j+1}(t, \lambda)=0 .
$$

Since the series in (1.33) converges uniformly we have

$$
\lim _{\substack{\lambda \rightarrow 0 \\ \operatorname{Im} \lambda \leqslant 0}} \widetilde{Z}_{\lambda}(t)=0 .
$$

The fact that $\lim _{\operatorname{Im} \rightarrow \underset{\lambda}{\lambda \leqslant 0}} \widetilde{Z}_{\lambda}^{\prime}(t)=0$ follows similarly.

We now study the case $b=0$ and $A(t)$ recessive. As before, we need a technical lemma.

Lemma 1.23. Assume ( $\mathrm{H} 1)$ to $(\mathrm{H} 6)$ with $b=0$ and $A(t)$ recessive, let

$$
C(0)=\sqrt{\frac{\pi}{2}} e^{-i \frac{\pi}{4}},
$$

and let

$$
\begin{aligned}
\Theta_{\lambda}(t)= & C(0) \mathcal{H}_{0}^{(2)}(\lambda t) \lambda^{-\frac{1}{2}} e^{i \lambda t}-t^{\frac{1}{2}}\left(C_{1}(0) \log \frac{2}{\lambda}+C_{2}(0)-C_{1}(0) \log t\right) \\
& +\int_{t}^{+\infty} \frac{k_{1}(t, s, \lambda)-k(t, s)}{\left(\log \frac{2}{\lambda}\right)^{-1}}\left[C_{1}(0) W_{1}(s)+\right. \\
& \left.+\left(\log \frac{2}{\lambda}\right)^{-1}\left(C_{2}(0) W_{1}(s)-C_{1}(0) W_{2}(s)\right)\right] d s
\end{aligned}
$$

Then for any $t \geqslant 1$, uniformly in $\operatorname{Im} \lambda \leqslant 0$,

$$
\left|\Theta_{\lambda}(t)\right| \leqslant\left\{\begin{array}{l}
c t^{\frac{1}{2}} \log t \text { for }|\lambda| t \geqslant 1 \\
c t^{\frac{1}{2}} \text { for }|\lambda| t \leqslant 1
\end{array}\right.
$$


Also for any fixed $t \geqslant 1$, we have

$$
\lim _{\substack{\lambda \rightarrow 0 \\ \operatorname{Im} \lambda \leqslant 0}} \Theta_{\lambda}(t)=0
$$

and

$$
\lim _{\substack{\lambda \rightarrow 0 \\ \operatorname{Im} \lambda \leqslant 0}} \Theta_{\lambda}^{\prime}(t)=0
$$

Proof. Since for $|\lambda| t \leqslant 1$

$$
\mathcal{H}_{0}^{(2)}(\lambda t)=\frac{2 i}{\pi} \sqrt{\lambda t}\left(\log \frac{2}{\lambda}-\log t\right)+\left(1-\frac{2 \gamma i}{\pi}\right) \sqrt{\lambda t}+O\left((\lambda t)^{\frac{3}{2}}\right)
$$

we have

$$
\begin{aligned}
& C(0) \mathcal{H}_{0}^{(2)}(\lambda t) \lambda^{-\frac{1}{2}} e^{i \lambda t}-t^{\frac{1}{2}}\left(C_{1}(0) \log \frac{2}{\lambda}+C_{2}(0)-C_{1}(0) \log t\right) \\
& =O\left(\lambda t^{\frac{3}{2}}\right) \log \frac{2}{|\lambda t|}=O\left(t^{\frac{1}{2}}\right) .
\end{aligned}
$$

In particular the above quantity vanishes as $\lambda \rightarrow 0$.

In the case $|\lambda t| \geqslant 1$, the standard estimates on the Hankel function give

$$
\begin{aligned}
& \left|C(0) \mathcal{H}_{0}^{(2)}(\lambda t) \lambda^{-\frac{1}{2}} e^{i \lambda t}-t^{\frac{1}{2}}\left(C_{1}(0) \log \frac{2}{\lambda}+C_{2}(0)-C_{1}(0) \log t\right)\right| \\
& \leqslant\left|C(0) \mathcal{H}_{0}^{(2)}(\lambda t) \lambda^{-\frac{1}{2}} e^{i \lambda t}\right|+t^{\frac{1}{2}}\left(\left|C_{1}(0)\right| \log \frac{2}{\lambda}+\left|C_{2}(0)\right|+\left|C_{1}(0)\right| \log t\right) \\
& \leqslant c t^{\frac{1}{2}} \log t
\end{aligned}
$$

We now estimate the integral part in the definition of $\Theta_{\lambda}(t)$. When $|\lambda t|<1$ we estimate separately the integral on $(t, 1 /|\lambda|)$ and the integral on $(1 /|\lambda|,+\infty)$.

The standard expansions for $\mathcal{H}_{0}^{(2)}$ and $\mathcal{J}_{0}$ give for $|\lambda t| \leqslant|\lambda s| \leqslant 1$

$$
\left|\frac{k_{1}(t, s, \lambda)-k(t, s)}{\left(\log \frac{2}{\lambda}\right)^{-1}}\right| \leqslant c|\lambda| \log \frac{2}{|\lambda|} t^{\frac{1}{2}} s^{\frac{3}{2}} \log \frac{2 s}{t}|\zeta(s)|
$$


so that

$$
\begin{aligned}
& \int_{t}^{1 /|\lambda|}\left|\frac{k_{1}(t, s, \lambda)-k(t, s)}{\left(\log \frac{2}{\lambda}\right)^{-1}}\right|\left|C_{1}(0) W_{1}(s)+\frac{C_{2}(0) W_{1}(s)-C_{1}(0) W_{2}(s)}{\log \frac{2}{\lambda}}\right| d s \\
& \leqslant c \int_{t}^{1 /|\lambda|} c|\lambda| \log \frac{2}{|\lambda|} t^{\frac{1}{2}} s^{\frac{3}{2}} \log \frac{2 s}{t}|\zeta(s)|\left[s^{\frac{1}{2}}+\left(\log \frac{2}{|\lambda|}\right)^{-1} s^{\frac{1}{2}} \log s\right] d s \\
& \leqslant c t^{\frac{1}{2}} \int_{t}^{1 /|\lambda|}|\zeta(s)| s \log ^{2} s d s .
\end{aligned}
$$

Since

$$
\frac{k_{1}(t, s, \lambda)-k(t, s)}{\left(\log \frac{2}{\lambda}\right)^{-1}} \rightarrow 0
$$

as $\lambda \rightarrow 0$ in $\operatorname{Im} \lambda \leqslant 0$ and $\zeta(s)$ is integrable in $s \log ^{2} s d s$, the dominated convergence theorem shows that the integral on the interval $(t, 1 /|\lambda|)$ vanishes as $\lambda \rightarrow 0$ in $\operatorname{Im} \lambda \leqslant 0$.

When $|\lambda s| \geqslant 1$ by (1.30) we have

$$
\begin{aligned}
\left|k_{1}(t, s, \lambda)-k(t, s)\right| & \leqslant\left|k_{1}(t, s, \lambda)\right|+|k(t, s)| \\
& \leqslant C|\lambda|^{-\frac{1}{2}} t^{\frac{1}{2}} \log \left(\frac{2}{|\lambda t|}\right)|\zeta(s)|+t^{\frac{1}{2}} s^{\frac{1}{2}} \log s|\zeta(s)| \\
& \leqslant C t^{\frac{1}{2}}\left(|\lambda|^{-\frac{1}{2}} \log \left(\frac{2}{|\lambda t|}\right)+s^{\frac{1}{2}} \log s\right)|\zeta(s)| \\
& \leqslant C t^{\frac{1}{2}} s^{\frac{1}{2}} \log s|\zeta(s)|
\end{aligned}
$$

and therefore

$$
\begin{aligned}
& \int_{1 /|\lambda|}^{+\infty}\left|\frac{k_{1}(t, s, \lambda)-k(t, s)}{\left(\log \frac{2}{\lambda}\right)^{-1}}\left[C_{1}(0) W_{1}(s)+\frac{C_{2}(0) W_{1}(s)-C_{1}(0) W_{2}(s)}{\log \frac{2}{\lambda}}\right]\right| d s \\
& \leqslant C t^{\frac{1}{2}} \int_{1 / \lambda \mid}^{+\infty}|\zeta(s)| s \log ^{2} s d s
\end{aligned}
$$

that vanishes as $\lambda \rightarrow 0$. It follows that $\lim _{\operatorname{Im} \lambda \leqslant 0} \Theta_{\lambda}(t)=0$. The fact that $\lim _{\operatorname{Im} \lambda \leqslant 0} \Theta_{\lambda}^{\prime}(t)=0$ follows similarly. 
In the case $|\lambda t| \geqslant 1$ the integral part in the definition of $\Theta_{\lambda}(t)$ is bounded by

$$
c t^{\frac{1}{2}} \int_{t}^{+\infty}|\zeta(s)| s \log ^{2} s d s
$$

The estimates on $\Theta_{\lambda}(t)$ follow.

Proof of Theorem 1.21. The proof follows the same lines as in the proof of Theorem 1.20. In this case we define

$$
\widetilde{Z}_{\lambda}(t)=\frac{\widetilde{v}_{\lambda}(t)-C_{1}(0) W_{1}(t)+\left(\log \frac{2}{\lambda}\right)^{-1}\left(C_{2}(0) W_{1}(t)-C_{1}(0) W_{2}(t)\right)}{\left(\log \frac{2}{\lambda}\right)^{-1}}
$$

and we apply Theorem A.3 to the equation

$$
\widetilde{Z}_{\lambda}(t)=\Theta_{\lambda}(t)+\int_{t}^{+\infty} \frac{k_{1}(t, s, \lambda)}{\zeta(s) s \log ^{2} s} \zeta(s) s \log ^{2} s \widetilde{Z}_{\lambda}(s) d s .
$$

For the kernel we have the estimates (see (1.31)

$$
\left|\frac{k_{1}(t, s, \lambda)}{\zeta(s) s \log ^{2} s}\right| \leqslant P_{0}(t) Q(s)
$$

and

$$
\left|\frac{\frac{\partial k_{1}}{\partial t}(t, s, \lambda)}{\zeta(s) s \log ^{2} s}\right| \leqslant P_{1}(t) Q(s)
$$

where $Q(s)=C \frac{\langle\lambda s\rangle^{1 / 2}\left|\log (2 s)\left(\log \frac{2}{|\lambda|}\right)^{-1}\right\rangle}{\left|s \log ^{2} s\right|}, P_{0}(t)=|\lambda|^{-1}\langle\lambda t\rangle^{1 / 2} \log \frac{2}{\langle\lambda t\rangle}$ and $P_{1}(t)=$ $\langle\lambda t\rangle^{-1 / 2} \log \frac{2}{\langle\lambda t\rangle}$. A simple computation shows that $\kappa \leqslant c|\lambda|^{\frac{1}{2}}\left(\log \frac{2}{|\lambda|}\right)^{-1}$ and $\kappa_{0}=c$. The proof follows as in the case $-\frac{1}{2}<b<0$.

\section{The Harish-Chandra function}

Since for $\lambda \in \mathbb{C}^{*}$

$$
\mathcal{W}\left(\Phi_{\lambda}, \Phi_{-\lambda}\right) \neq 0
$$

the eigenfunction $\varphi_{\lambda}$ is a linear combination of $\Phi_{\lambda}$ and $\Phi_{-\lambda}$. We define the HarishChandra function $c(\lambda)$ by

$$
\varphi_{\lambda}(t)=c(\lambda) \Phi_{\lambda}(t)+c(-\lambda) \Phi_{-\lambda}(t) .
$$


By Corollary 1.18 we have

$$
\begin{aligned}
& \mathcal{W}\left(A^{1 / 2}(t) \varphi_{\lambda}(t), A^{1 / 2}(t) \Phi_{-\lambda}(t)\right) \\
& =c(\lambda) \mathcal{W}\left(A^{1 / 2}(t) \Phi_{\lambda}(t), A^{1 / 2}(t) \Phi_{-\lambda}(t)\right) \\
& =-2 i \lambda c(\lambda),
\end{aligned}
$$

so that

$$
c(\lambda)=i \frac{\mathcal{W}\left(A^{1 / 2}(t) \varphi_{\lambda}(t), A^{1 / 2}(t) \Phi_{-\lambda}(t)\right)}{2 \lambda} .
$$

Since the Wronskian is independent of $t$ we also have

$$
c(\lambda)=i \frac{\lim _{t \rightarrow \infty} \mathcal{W}\left(A^{1 / 2}(t) \varphi_{\lambda}(t), A^{1 / 2}(t) \Phi_{-\lambda}(t)\right)}{2 \lambda} .
$$

In the next theorem we obtain an asymptotic expansion for $c(\lambda)$ based on the asymptotic expansion for $A^{1 / 2}(t) \varphi_{\lambda}(t)$.

Theorem 2.1. Assume (H1) to (H4) be satisfied and let $G$ and $a_{m}$ be defined respectively by (0.6) and Lemma 1.1. Let $M \geqslant 0$ and assume $G^{(k)} \in L^{1}((1,+\infty), d t)$ for $k=0, \ldots, M$. Then, for every $\lambda \in \mathbb{C}^{*}, \operatorname{Im}(\lambda) \leqslant 0$ we have

$$
c(\lambda)=\sqrt{\frac{1}{2 \pi}} \sum_{m=0}^{M} a_{m}(\infty) \frac{e^{i\left(-\frac{\pi}{2}(\alpha+m)-\frac{\pi}{4}\right)}}{\lambda^{m+\alpha+1 / 2}}+E_{M}(\lambda),
$$

where

$$
\left|E_{M}(\lambda)\right| \leqslant C \frac{e^{\frac{C}{|\lambda|}}}{|\lambda|^{M+\alpha+3 / 2}}
$$

and

$$
a_{m}(\infty)=\lim _{t \rightarrow+\infty} a_{m}(t) .
$$

Proof. By Theorem 1.2 and Theorem 1.17 we have

$$
\begin{aligned}
& \lim _{t \rightarrow+\infty} \mathcal{W}\left(A^{1 / 2}(t) \varphi_{\lambda}(t), A^{1 / 2}(t) \Phi_{-\lambda}(t)\right) \\
= & \lim _{t \rightarrow+\infty} \mathcal{W}\left(\sum_{m=0}^{M} \frac{a_{m}(t)}{\lambda^{m+\alpha+1 / 2}} \mathcal{J}_{\alpha+m}(\lambda t)+R_{M}(\lambda, t), e^{-i \lambda t}+e^{-i \lambda t} \mathcal{R}(-\lambda, t)\right) \\
= & \sum_{m=0}^{M} \lim _{t \rightarrow+\infty} \mathcal{W}\left(\frac{a_{m}(t)}{\lambda^{m+\alpha+1 / 2}} \mathcal{J}_{\alpha+m}(\lambda t), e^{-i \lambda t}\right) \\
& +\sum_{m=0}^{M} \lim _{t \rightarrow+\infty} \mathcal{W}\left(\frac{a_{m}(t)}{\lambda^{m+\alpha+1 / 2}} \mathcal{J}_{\alpha+m}(\lambda t), e^{-i \lambda t} \mathcal{R}(-\lambda, t)\right) \\
& +\lim _{t \rightarrow+\infty} \mathcal{W}\left(R_{M}(\lambda, t), e^{-i \lambda t}\right)+\lim _{t \rightarrow+\infty} \mathcal{W}\left(R_{M}(\lambda, t), e^{-i \lambda t} \mathcal{R}(-\lambda, t)\right)
\end{aligned}
$$


Let us begin with the principal part. By Lemma $1.1 a_{m}^{\prime}, a_{m}^{\prime \prime} \in L^{1}((1,+\infty), d t)$. This easily implies $a_{m}^{\prime}(t) \rightarrow 0$ as $t \rightarrow+\infty$, so that

$$
\begin{aligned}
& \frac{1}{\lambda^{m+\alpha+1 / 2}} \lim _{t \rightarrow \infty} \mathcal{W}\left(a_{m}(t) \mathcal{J}_{\alpha+m}(\lambda t), e^{-i \lambda t}\right) \\
= & \frac{1}{\lambda^{m+\alpha+1 / 2}} \lim _{t \rightarrow \infty}\left\{a_{m}(t) \mathcal{J}_{\alpha+m}(\lambda t)(-i \lambda) e^{-i \lambda t}-a_{m}^{\prime}(t) \mathcal{J}_{\alpha+m}(\lambda t) e^{-i \lambda t}\right. \\
& \left.-a_{m}(t)\left(\mathcal{J}_{\alpha+m}(\lambda t)\right)^{\prime} e^{-i \lambda t}\right\} \\
= & \frac{1}{\lambda^{m+\alpha+1 / 2}} \lim _{t \rightarrow \infty} a_{m}(t) e^{-i \lambda t}\left(\mathcal{J}_{\alpha+m}(\lambda t)(-i \lambda)-\left(\mathcal{J}_{\alpha+m}(\lambda t)\right)^{\prime}\right)
\end{aligned}
$$

The standard expansions for $\mathcal{J}_{\alpha+m}(\lambda t)$ and the well known identity

$$
\left(\mathcal{J}_{\alpha+m}(\lambda t)\right)^{\prime}=\frac{\left(\frac{1}{2}-\alpha-m\right)}{t} \mathcal{J}_{\alpha+m}(\lambda t)+\lambda \mathcal{J}_{\alpha+m-1}(\lambda t)
$$

give

$$
\lim _{t \rightarrow+\infty} \mathcal{W}\left(\frac{a_{m}(t)}{\lambda^{m+\alpha+1 / 2}} \mathcal{J}_{\alpha+m}(\lambda t), e^{-i \lambda t}\right)=-\sqrt{\frac{2}{\pi}} \frac{e^{i\left(-\frac{\pi}{2}(\alpha+m)+\frac{\pi}{4}\right)}}{\lambda^{m+\alpha-1 / 2}} \lim _{t \rightarrow+\infty} a_{m}(t) .
$$

Observe that $\lim _{t \rightarrow+\infty} a_{m}(t)$ exists since $a_{m}^{\prime} \in L^{1}((1,+\infty), d t)$. The remainder terms

$$
\mathcal{W}\left(\frac{a_{m}(t)}{\lambda^{m+\alpha+1 / 2}} \mathcal{J}_{\alpha+m}(\lambda t), e^{-i \lambda t} \mathcal{R}(-\lambda, t)\right)
$$

and

$$
\mathcal{W}\left(R_{M}(\lambda, t), e^{-i \lambda t} \mathcal{R}(-\lambda, t)\right)
$$

tend to 0 as $t \rightarrow+\infty$, as the functions $\frac{a_{m}(t)}{\lambda^{m+\alpha+1 / 2}} \mathcal{J}_{\alpha+m}(\lambda t)$ and their derivatives are all bounded by $c e^{|\operatorname{Im} \lambda| t}$ (and so do $R_{M}(\lambda, t)$ and its derivative) while $e^{-i \lambda t} \mathcal{R}(-\lambda, t)$ and its derivative are bounded by $e^{\operatorname{Im}(\lambda) \mathrm{t}}$ times a term that tends to 0 as $t \rightarrow+\infty$.

As for the other term, by Theorem 1.2

$$
\lim _{t \rightarrow+\infty}\left|\mathcal{W}\left(R_{M}(\lambda, t), e^{-i \lambda t}\right)\right| \leqslant \frac{C e^{\frac{C}{\lambda \mid}}}{|\lambda|^{M+\alpha+1 / 2}} .
$$

By (2.3) the theorem follows.

In the sequel we will need the following expansion for $|c(\lambda)|^{2}$. 
Theorem 2.2. Assume (H1) to (H4) be satisfied. Let $M \geqslant 0$ and assume $G^{(k)} \in$ $L^{1}((1,+\infty), d t)$ for $k=0, \ldots, 2 M+1$. Then for every $\lambda>0$ we have

$$
|c(\lambda)|^{2}=\frac{1}{2 \pi \lambda^{2 \alpha+1}} \sum_{s=0}^{M} \frac{d_{s}}{\lambda^{2 s}}+\widetilde{E}_{2 M+1}(\lambda),
$$

where

$$
d_{s}=(-1)^{s} \sum_{m=0}^{2 s}(-1)^{m} a_{m}(\infty) a_{2 s-m}(\infty)
$$

and

$$
\left|\widetilde{E}_{2 M+1}(\lambda)\right| \leqslant C \frac{e^{\frac{C}{\lambda \mid}}}{|\lambda|^{2 M+2 \alpha+3}} .
$$

Furthermore, if $G, G^{\prime} \in L^{1}((1,+\infty), d t)$ then for every $\lambda \in \mathbb{C}^{*}, \operatorname{Im}(\lambda) \leqslant 0$, we have

$$
|c(\lambda)|^{2}=\frac{a_{0}^{2}}{2 \pi|\lambda|^{2 \alpha+1}}+\frac{a_{1}(\infty) a_{0}}{\pi|\lambda|^{2 \alpha+1}} \operatorname{Im} \frac{1}{\lambda}+\widetilde{\mathrm{E}}_{1}(\lambda),
$$

and

$$
\left|\widetilde{E}_{1}(\lambda)\right| \leqslant C \frac{e^{\frac{C}{|\lambda|}}}{|\lambda|^{2 \alpha+3}}
$$

Finally, if $G \in L^{1}((1,+\infty), d t)$ then for every $\lambda \in \mathbb{C}^{*}, \operatorname{Im}(\lambda) \leqslant 0$, we have

$$
|c(\lambda)|^{2}=\frac{a_{0}^{2}}{2 \pi|\lambda|^{2 \alpha+1}}+\widetilde{E}(\lambda),
$$

and

$$
|\widetilde{E}(\lambda)| \leqslant C \frac{e^{\frac{C}{|\lambda|}}}{|\lambda|^{2 \alpha+2}}
$$

Proof. By Theorem 2.1

$$
\begin{aligned}
c(\lambda) \overline{c(\lambda)}= & \frac{1}{2 \pi}\left(\sum_{m=0}^{2 M+1} \frac{e^{i\left(-\frac{\pi}{2}(\alpha+m)-\frac{\pi}{4}\right)}}{\lambda^{m+\alpha+\frac{1}{2}}} a_{m}(\infty)+E_{2 M+1}(\lambda)\right) \\
& \times\left(\sum_{n=0}^{2 M+1} \frac{e^{i\left(\frac{\pi}{2}(\alpha+n)+\frac{\pi}{4}\right)}}{\lambda^{n+\alpha+\frac{1}{2}}} a_{n}(\infty)+\overline{E_{2 M+1}(\lambda)}\right) \\
= & \frac{1}{2 \pi} \sum_{s=0}^{2 M+1} \sum_{m=0}^{s} \frac{e^{i \frac{\pi}{2}(s-2 m)}}{\lambda^{s+2 \alpha+1}} a_{m}(\infty) a_{s-m}(\infty)+\widetilde{E}_{2 M+1}(\lambda) \\
= & \frac{1}{2 \pi} \sum_{s=0}^{2 M+1} \frac{i^{s}}{\lambda^{s+2 \alpha+1}} \sum_{m=0}^{s}(-1)^{m} a_{m}(\infty) a_{s-m}(\infty)+\widetilde{E}_{2 M+1}(\lambda) .
\end{aligned}
$$


Since for $s$ odd, $\sum_{m=0}^{s}(-1)^{m} a_{m}(\infty) a_{s-m}(\infty)=0$ we have

$$
\begin{aligned}
|c(\lambda)|^{2} & =\frac{1}{2 \pi} \sum_{s=0}^{M} \frac{(-1)^{s}}{\lambda^{2 s+2 \alpha+1}} \sum_{m=0}^{2 s}(-1)^{m} a_{m}(\infty) a_{2 s-m}(\infty)+\widetilde{E}_{2 M+1}(\lambda) \\
& =\frac{1}{2 \pi} \sum_{s=0}^{M} \frac{1}{\lambda^{2 s+2 \alpha+1}} d_{s}+\widetilde{E}_{2 M+1}(\lambda)
\end{aligned}
$$

The estimate for $\widetilde{E}_{2 M+1}(\lambda)$ follows from the analogous estimate for the remainder in Theorem 2.1. The rest of the theorem follows in a similar way.

Corollary 2.3. Assume (H1) to (H4) and let $G \in L^{1}((1,+\infty), d t)$. Then if $|\lambda| \rightarrow$ $+\infty, \operatorname{Im} \lambda \leqslant 0$ one has

$$
\frac{1}{|c(\lambda)|} \approx|\lambda|^{\alpha+\frac{1}{2}} .
$$

Theorem 2.4. Assume (H1) to (H6) hold for some $b>-1 / 2$. If $A$ is non singular (see Remark 1.15) then if $\lambda \rightarrow 0, \operatorname{Im} \lambda \leqslant 0$

$$
\frac{1}{|c(\lambda)|} \approx|\lambda|^{b+\frac{1}{2}}
$$

and $\lambda^{-b-\frac{1}{2}} c(\lambda)^{-1}$ is analytic in $\operatorname{Im} \lambda<0$ and continuous in $\operatorname{Im} \lambda \leqslant 0$. If $A(t)$ is singular then if $\lambda \rightarrow 0, \operatorname{Im} \lambda \leqslant 0$

$$
\frac{1}{|c(\lambda)|} \approx|\lambda|^{1 / 2}\left(\log \frac{2}{|\lambda|}\right)^{-1}
$$

and $\lambda^{-1 / 2} \log \frac{2}{\lambda} c(\lambda)^{-1}$ is analytic in $\operatorname{Im} \lambda<0$ and continuous in $\operatorname{Im} \lambda \leqslant 0$.

Remark 2.5. When $b>0$, or $b=0$ and $A(t)$ is singular the above result is due to Bloom and $\mathrm{Xu}$ (see [2, Proposition 3.16]). Using the expansions of $\Phi_{-\lambda}$ proved in the previous section we complete their arguments in order to obtain the estimates for $b>-\frac{1}{2}$. For the sake of completeness we give the proof in all cases.

Proof. Using the method of successive approximations it is not difficult to prove that $\varphi_{\lambda}$ and $\varphi_{\lambda}^{\prime}$ are entire functions in $\lambda$. By Theorem 1.19 and equation (2.2) it follows that $c(\lambda)$ is continuous for $\operatorname{Im} \lambda \leqslant 0, \lambda \neq 0$ and analytic in $\operatorname{Im} \lambda<0$.

We now prove that $c(\lambda) \neq 0$ for $\lambda \neq 0$ and $\operatorname{Im} \lambda \leqslant 0$. Observe that since $\varphi_{\lambda} \neq \equiv 0$, then $c(\lambda)$ and $c(-\lambda)$ cannot vanish simultaneously. Hence if $\lambda \in \mathbb{R}$, since $c(\lambda)=\overline{c(-\lambda)}$ we have $c(\lambda) \neq 0$. 
Assume now $\lambda=\xi+i \eta, \eta<0$, by Theorem 1.17 we have $\Phi_{-\lambda}, \Phi_{-\lambda}^{\prime} \in$ $L^{2}\left(\mathbb{R}^{+}, A(t) d t\right)$. Thus

$$
\begin{aligned}
& \int_{0}^{+\infty}\left(\lambda^{2}+\rho^{2}\right)\left|\Phi_{-\lambda}(t)\right|^{2} A(t) d t \\
& =-\int_{0}^{+\infty} \overline{\Phi_{-\lambda}(t)} \mathcal{L} \Phi_{-\lambda}(t) A(t) d t=-\int_{0}^{+\infty} \overline{\Phi_{-\lambda}(t)}\left(\Phi_{-\lambda}^{\prime}(t) A(t)\right)^{\prime} d t \\
& =-\left[\overline{\Phi_{-\lambda}(t)} \Phi_{-\lambda}^{\prime}(t) A(t)\right]_{0}^{+\infty}+\int_{0}^{+\infty}\left|\Phi_{-\lambda}^{\prime}(t)\right|^{2} A(t) d t
\end{aligned}
$$

If $c(\lambda)=0$ then $\varphi_{\lambda}=c(-\lambda) \Phi_{-\lambda}$ and therefore $\lim _{t \rightarrow 0^{+}} \overline{\Phi_{-\lambda}(t)} \Phi_{-\lambda}^{\prime}(t) A(t)=$ 0 . Also by Theorem $1.17 \lim _{t \rightarrow+\infty} \overline{\Phi_{-\lambda}(t)} \Phi_{-\lambda}^{\prime}(t) A(t)=0$. It follows that $\left(\lambda^{2}+\rho^{2}\right) \geqslant 0$ and therefore $c(\lambda) \neq 0$ for $\xi \neq 0$ or $\eta<-\rho$.

Assume now $-\rho \leqslant \eta<0$ and observe that by Theorem 1.17 and (2.1) we have

$$
c(i \eta)=\lim _{t \rightarrow+\infty} e^{\eta t} \sqrt{A(t)} \varphi_{i \eta}(t) .
$$

Since $A(t) \geqslant C e^{2 \rho t}$ with $C>0$, by Lemma 1.4 we have

$$
c(i \eta) \geqslant C>0 \text {. }
$$

We now assume $A(t)$ dominant and we set

$$
c_{1}(\lambda)= \begin{cases}\lambda^{a+\frac{1}{2}} c(\lambda) & b>0 \\ \lambda^{\frac{1}{2}}\left(\log \frac{2}{\lambda}\right)^{-1} c(\lambda) & b=0 .\end{cases}
$$

By (2.2) we have

$$
c_{1}(\lambda)=\frac{i}{2} \mathcal{W}\left(\sqrt{A} \varphi_{\lambda}, \sqrt{A} \widetilde{\Phi}_{-\lambda}\right) .
$$

By Theorem 1.19 we know that $c_{1}(\lambda)$ is continuous in $\operatorname{Im} \lambda \leqslant 0$ and analytic in $\operatorname{Im} \lambda<0$. It follows that

$$
\lim _{\substack{\lambda \rightarrow 0 \\ \operatorname{Im} \lambda \leqslant 0}} c_{1}(\lambda)=c_{1}(0)
$$

exists. Being $A(t)$ dominant $\sqrt{A} \varphi_{0}$ and $W_{1}$ behave differently at infinity and therefore they are linearly independent. Since

$$
c_{1}(0)=\frac{i}{2} \mathcal{W}\left(\sqrt{A} \varphi_{0}, C_{1}(a) W_{1}\right)
$$

we have $c_{1}(0) \neq 0$ and in this case we obtain the required estimates for $\frac{1}{|c(\lambda)|}$. 
Assume $A(t)$ recessive, so that $\rho=0$ and $-\frac{1}{2}<b \leqslant 0$ and $\sqrt{A(t)}=$ $\sqrt{A(t)} \varphi_{0}(t)=c W_{1}(t)$. Consider first $-\frac{1}{2}<b<0$. Since $\lambda \mapsto \varphi_{\lambda}$ is even we have $\varphi_{\lambda}=1+O\left(\lambda^{2}\right)$, also $\varphi_{\lambda}^{\prime}=O\left(\lambda^{2}\right)$. Applying Lemma 1.20 we have

$$
\begin{aligned}
\mathcal{W}\left(\sqrt{A} \varphi_{\lambda}, \sqrt{A} \widetilde{\Phi}_{-\lambda}\right) & =\mathcal{W}\left(c W_{1}+O\left(\lambda^{2}\right), C_{1}(a) W_{1}+C_{2}(a) \lambda^{2 a} W_{2}+\lambda^{2 a} Z_{\lambda}\right) \\
& =\lambda^{2 a} c C_{2}(a) \mathcal{W}\left(W_{1}, W_{2}\right)+o\left(|\lambda|^{2 a}\right)
\end{aligned}
$$

since

$$
\lim _{\substack{\lambda \rightarrow 0 \\ \operatorname{Im} \lambda \leqslant 0}} Z_{\lambda}(t)=0
$$

and

$$
\lim _{\substack{\lambda \rightarrow 0 \\ \operatorname{Im} \lambda \leqslant 0}} \frac{d}{d t} Z_{\lambda}(t)=0 .
$$

Therefore

$$
c(\lambda)=\lambda^{a-\frac{1}{2}}\left(c C_{2}(a) \frac{i}{2} \mathcal{W}\left(W_{1}, W_{2}\right)+o(1)\right)
$$

and since $W_{1}$ and $W_{2}$ are linearly independent we have

$$
|c(\lambda)| \approx|\lambda|^{-b-\frac{1}{2}} .
$$

It remains to consider the case $A(t)$ recessive and $b=0$.

Applying Theorem 1.21 we have

$$
\begin{aligned}
& \mathcal{W}\left(\sqrt{A} \varphi_{\lambda}, \sqrt{A} \widetilde{\Phi}_{-\lambda}\right) \\
& =\mathcal{W}\left(c W_{1}+O\left(\lambda^{2}\right), C_{1}(0) W_{1}+\left(\log \frac{2}{\lambda}\right)^{-1}\left(C_{2}(0) W_{1}-C_{1}(0) W_{2}\right)+\left(\log \frac{2}{\lambda}\right)^{-1} Z_{\lambda}\right) \\
& =-C_{1}(0) c\left(\log \frac{2}{\lambda}\right)^{-1} \mathcal{W}\left(W_{1}, W_{2}\right)+o\left(\left(\log \frac{2}{\lambda}\right)^{-1}\right)
\end{aligned}
$$

since

$$
\lim _{\substack{\lambda \rightarrow 0 \\ \operatorname{Im} \lambda \leqslant 0}} Z_{\lambda}(t)=0
$$

and

$$
\lim _{\substack{\lambda \rightarrow 0 \\ \operatorname{Im} \lambda \leqslant 0}} \frac{d}{d t} Z_{\lambda}(t)=0 .
$$

Therefore

$$
c(\lambda)=\lambda^{-\frac{1}{2}}\left(-\frac{i}{2} C_{1}(0) c \mathcal{W}\left(W_{1}, W_{2}\right)+o(1)\right)
$$


and since $W_{1}$ and $W_{2}$ are linearly independent we have

$$
|c(\lambda)| \approx|\lambda|^{-\frac{1}{2}} .
$$

\section{Convergence and divergence results}

Let us define the operators.

$$
\widetilde{S}_{R} f=A^{\frac{1}{2}} S_{R}\left(f A^{-\frac{1}{2}}\right)
$$

and

$$
\widetilde{F}_{R} f=A^{\frac{1}{2}} F_{R}\left(f A^{-\frac{1}{2}}\right) .
$$

A simple computation shows that

$$
\begin{aligned}
& \widetilde{S}_{R} f(x)=\int_{0}^{+\infty} \widetilde{S}_{R}(x, t) f(t) d t \\
& \widetilde{F}_{R} f(x)=\int_{0}^{+\infty} \widetilde{F}_{R}(x, t) f(t) d t,
\end{aligned}
$$

with

$$
\widetilde{S}_{R}(x, t)=\int_{0}^{R} \sqrt{A(t)} \varphi_{\lambda}(t) \sqrt{A(x)} \varphi_{\lambda}(x) \frac{d \lambda}{2 \pi|c(\lambda)|^{2}}
$$

and

$$
\widetilde{F}_{R}(x, t)=\int_{0}^{R} \mathcal{J}_{\alpha}(\lambda t) \mathcal{J}_{\alpha}(\lambda x) d \lambda .
$$

In the next lemma we estimate the difference of the kernels of the above two operators. This is the main step to prove Theorem 0.2.

Lemma 3.1. Assume that $A(t)$ satisfies $(\mathrm{H} 1)$ to $(\mathrm{H} 6)$ and let $\zeta^{\prime}(t) \in L^{1}((1,+\infty), d t)$. Let $\eta>0$. Then there exists a positive constant $c_{\eta}$ such that

$$
\left|\widetilde{S}_{R}(x, t)-\widetilde{F}_{R}(x, t)\right| \leqslant \begin{cases}\frac{c_{\eta}}{1+R|x-t|} & \text { for } x>\eta \text { or } t>\eta, \\ c_{\eta} & \text { for } x \leqslant \eta \text { and } t \leqslant \eta .\end{cases}
$$

Proof. Assume first $x>\eta$ or $t>\eta$. Since both kernels are symmetric it is enough to consider $x>\eta$ and $x>t$. Since

$$
\varphi_{\lambda}(x)=c(\lambda) \Phi_{\lambda}(x)+c(-\lambda) \Phi_{-\lambda}(x)
$$


and for $\lambda$ real $c(-\lambda)=\overline{c(\lambda)}$ we have

$$
\begin{aligned}
\tilde{S}_{R}(x, t)= & \int_{0}^{R} \sqrt{A(t)} \varphi_{\lambda}(t) \sqrt{A(x)} c(\lambda) \Phi_{\lambda}(x) \frac{d \lambda}{2 \pi|c(\lambda)|^{2}} \\
& +\int_{0}^{R} \sqrt{A(t)} \varphi_{\lambda}(t) \sqrt{A(x)} c(-\lambda) \Phi_{-\lambda}(x) \frac{d \lambda}{2 \pi|c(\lambda)|^{2}} \\
= & \int_{-R}^{R} \sqrt{A(t)} \varphi_{\lambda}(t) \sqrt{A(x)} \Phi_{-\lambda}(x) \frac{d \lambda}{2 \pi c(\lambda)} .
\end{aligned}
$$

Since $\widetilde{F}_{R}(x, t)$ is a particular case of $\widetilde{S}_{R}(x, t)$ when $A(t)=t^{2 \alpha+1}$ we can write

$$
\widetilde{F}_{R}(x, t)=\int_{-R}^{R} \sqrt{t^{2 \alpha+1}} \boldsymbol{\varphi}_{\lambda}(t) \sqrt{x^{2 \alpha+1}} \boldsymbol{\Phi}_{-\lambda}(x) \frac{d \lambda}{2 \pi \mathbf{c}(\lambda)}
$$

where $\boldsymbol{\varphi}_{\lambda}(t)=2^{\alpha} \Gamma(\alpha+1) \frac{J_{\alpha}(\lambda t)}{(\lambda t)^{\alpha}}, \boldsymbol{\Phi}_{-\lambda}(x)=\sqrt{\frac{\pi \lambda}{2}} e^{-i \frac{\pi}{4}(2 \alpha+1)} x^{-\alpha} H_{\alpha}^{(2)}(\lambda x)$ and $\mathbf{c}(\lambda)=\frac{2^{\alpha} \Gamma(\alpha+1) e^{-i \frac{\pi}{4}(2 \alpha+1)}}{\sqrt{2 \pi} \lambda^{\alpha+\frac{1}{2}}}$. It follows that

$$
\begin{aligned}
& \widetilde{S}_{R}(x, t)-\widetilde{F}_{R}(x, t) \\
& =\int_{-R}^{R}\left(\sqrt{A(t)} \varphi_{\lambda}(t) \sqrt{A(x)} \frac{\Phi_{-\lambda}(x)}{2 \pi c(\lambda)}-\sqrt{t^{2 \alpha+1}} \varphi_{\lambda}(t) \sqrt{x^{2 \alpha+1}} \frac{\boldsymbol{\Phi}_{-\lambda}(x)}{2 \pi \mathbf{c}(\lambda)}\right) d \lambda .
\end{aligned}
$$

Observe now that $\varphi_{\lambda}(t)$ and $\varphi_{\lambda}(t)$ are entire functions in $\lambda$; that

$$
\frac{\Phi_{-\lambda}(t)}{c(\lambda)}=\frac{\lambda^{a-\frac{1}{2}} \Phi_{-\lambda}(t)}{\lambda^{b+\frac{1}{2}} c(\lambda)} \lambda^{b+1-a}
$$

is analytic for $\operatorname{Im} \lambda<0$ and continuous for $\operatorname{Im} \lambda \leqslant 0$ by Theorem 1.19 and 2.4 (the case $a=0$ being similar) and that $\frac{\Phi_{-\lambda}(x)}{\mathbf{c}(\lambda)}$ is analytic and continuous in the same domain. Therefore we can change the path of integration in the above integral. Let $\gamma=\left\{\operatorname{Re}^{i \theta}:-\pi \leqslant \theta \leqslant 0\right\}$. Then

$$
\begin{aligned}
& \widetilde{S}_{R}(x, t)-\widetilde{F}_{R}(x, t) \\
& =-\int_{\gamma}\left(\sqrt{A(t)} \varphi_{\lambda}(t) \sqrt{A(x)} \frac{\Phi_{-\lambda}(x)}{2 \pi c(\lambda)}-\sqrt{t^{2 \alpha+1}} \boldsymbol{\varphi}_{\lambda}(t) \sqrt{x^{2 \alpha+1}} \frac{\boldsymbol{\Phi}_{-\lambda}(x)}{2 \pi \mathbf{c}(\lambda)}\right) d \lambda .
\end{aligned}
$$

By Theorem 1.2 we have

$$
\sqrt{A(t)} \varphi_{\lambda}(t)=\sqrt{t^{2 \alpha+1}} \varphi_{\lambda}(t)+R_{0}(\lambda, t)
$$

where, uniformly in $|\lambda| \geqslant 1$,

$$
\left|R_{0}(\lambda, t)\right| \leqslant \frac{C}{|\lambda|^{\alpha+\frac{3}{2}}} e^{|\operatorname{Im} \lambda t|} .
$$


By Theorem 1.17, for $x>\eta$

$$
\begin{aligned}
& \sqrt{A(x)} \Phi_{-\lambda}(x)=e^{-i \lambda x}\left(1+\mathcal{R}_{1}(\lambda, x)\right) \\
& \sqrt{x^{2 \alpha+1}} \Phi_{-\lambda}(x)=e^{-i \lambda x}\left(1+\mathcal{R}_{2}(\lambda, x)\right)
\end{aligned}
$$

where

$$
\left|\mathcal{R}_{j}(\lambda, x)\right| \leqslant \frac{c_{\eta}}{|\lambda|}
$$

Finally, by Theorem 2.1,

$$
c(\lambda)^{-1}=\mathbf{c}(\lambda)^{-1}(1+E(\lambda))
$$

where, uniformly in $|\lambda| \geqslant 1$,

$$
|E(\lambda)| \leqslant \frac{C}{|\lambda|}
$$

Therefore

$$
\widetilde{S}_{R}(x, t)-\widetilde{F}_{R}(x, t)=\frac{-1}{2 \pi}\left(I_{1}+I_{2}+I_{3}+I_{4}\right)
$$

where

$$
\begin{aligned}
& I_{1}=\int_{\gamma} \sqrt{t^{2 \alpha+1}} \boldsymbol{\varphi}_{\lambda}(t) \frac{e^{-i \lambda x} \mathcal{R}_{1}(\lambda, x)}{\mathbf{c}(\lambda)} d \lambda \\
& I_{2}=\int_{\gamma} \sqrt{t^{2 \alpha+1}} \boldsymbol{\varphi}_{\lambda}(t) \frac{e^{-i \lambda x}\left(1+\mathcal{R}_{1}(\lambda, x)\right)}{\mathbf{c}(\lambda)} E(\lambda) d \lambda \\
& I_{3}=\int_{\gamma} R_{0}(\lambda, t) \frac{e^{-i \lambda x}\left(1+\mathcal{R}_{1}(\lambda, x)\right)}{\mathbf{c}(\lambda)}(1+E(\lambda)) d \lambda \\
& I_{4}=-\int_{\gamma} \sqrt{t^{2 \alpha+1}} \boldsymbol{\varphi}_{\lambda}(t) \frac{e^{-i \lambda x} \mathcal{R}_{2}(\lambda, x)}{\mathbf{c}(\lambda)} d \lambda .
\end{aligned}
$$

By the classical estimates for Bessel functions we have

$$
\begin{aligned}
\left|I_{1}\right| & \leqslant c_{\eta} \int_{-\pi}^{0} e^{R \sin \theta(x-t)} d \theta \\
& \leqslant \frac{c_{\eta}}{1+R|x-t|}
\end{aligned}
$$

Similar computations give the same bounds for $I_{2}, I_{3}$ and $I_{4}$.

We now consider the case $x \leqslant \eta$ and $t \leqslant \eta$. 
Let

$$
\begin{aligned}
& \left|\widetilde{S}_{R}(x, t)-\widetilde{F}_{R}(x, t)\right| \\
& =\left|\int_{0}^{R}\left(\sqrt{A(t)} \varphi_{\lambda}(t) \sqrt{A(x)} \varphi_{\lambda}(x) \frac{1}{2 \pi|c(\lambda)|^{2}}-\mathcal{J}_{\alpha}(\lambda t) \mathcal{J}_{\alpha}(\lambda x)\right) d \lambda\right| \\
& \leqslant\left|\int_{0}^{1} \ldots d \lambda\right|+\left|\int_{1}^{R} \ldots d \lambda\right| .
\end{aligned}
$$

For the first integral we have

$$
\begin{aligned}
& \left|\int_{0}^{1}\left[\sqrt{A(x)} \varphi_{\lambda}(x) \sqrt{A(t)} \varphi_{\lambda}(t) \frac{1}{2 \pi|c(\lambda)|^{2}}-\mathcal{J}_{\alpha}(\lambda x) \mathcal{J}_{\alpha}(\lambda t)\right] d \lambda\right| \\
& \leqslant \int_{0}^{1}\left|\sqrt{A(x)} \varphi_{\lambda}(x) \sqrt{A(t)} \varphi_{\lambda}(t)\right| \frac{1}{2 \pi|c(\lambda)|^{2}} d \lambda+\int_{0}^{1}\left|\mathcal{J}_{\alpha}(\lambda x) \mathcal{J}_{\alpha}(\lambda t)\right| d \lambda \\
& \leqslant C
\end{aligned}
$$

since $\left|\varphi_{\lambda}(x)\right| \leqslant 1$ and $|c(\lambda)|^{-2}$ is bounded for small $\lambda$ by Theorem 2.4.

Let us consider the second integral. When $\lambda>1$, by Theorem 1.2, we have

$$
\sqrt{A(t)} \varphi_{\lambda}(t)=\frac{a_{0}}{\lambda^{\alpha+1 / 2}} \mathcal{J}_{\alpha}(\lambda t)+\frac{a_{1}(t)}{\lambda^{\alpha+3 / 2}} \mathcal{J}_{\alpha+1}(\lambda t)+O\left(\lambda^{-\alpha-\frac{5}{2}}\right) .
$$

Also, by Theorem 2.2, we have

$$
\frac{1}{2 \pi|c(\lambda)|^{2}}=\frac{1}{a_{0}^{2}} \lambda^{2 \alpha+1}+O\left(\lambda^{2 \alpha-1}\right) .
$$

A simple computation then shows that

$$
\begin{aligned}
& \sqrt{A(t)} \varphi_{\lambda}(t) \sqrt{A(x)} \varphi_{\lambda}(x) \frac{1}{2 \pi|c(\lambda)|^{2}}-\mathcal{J}_{\alpha}(\lambda t) \mathcal{J}_{\alpha}(\lambda x) \\
& =\frac{1}{a_{0} \lambda}\left(a_{1}(x) \mathcal{J}_{\alpha}(\lambda t) \mathcal{J}_{\alpha+1}(\lambda x)+a_{1}(t) \mathcal{J}_{\alpha+1}(\lambda t) \mathcal{J}_{\alpha}(\lambda x)\right)+O\left(\lambda^{-2}\right) .
\end{aligned}
$$

We are reduced to showing that

$$
\left|\int_{1}^{R}\left(a_{1}(x) \mathcal{J}_{\alpha+1}(\lambda x) \mathcal{J}_{\alpha}(\lambda t)+a_{1}(t) \mathcal{J}_{\alpha+1}(\lambda t) \mathcal{J}_{\alpha}(\lambda x)\right) \frac{1}{\lambda} d \lambda\right| \leqslant C .
$$

Without loss of generality, assume $x \leqslant t$. Then

$$
\begin{aligned}
& \int_{1}^{\eta / x}\left|a_{1}(x) \mathcal{J}_{\alpha+1}(\lambda x) \mathcal{J}_{\alpha}(\lambda t)+a_{1}(t) \mathcal{J}_{\alpha+1}(\lambda t) \mathcal{J}_{\alpha}(\lambda x)\right| \frac{1}{\lambda} d \lambda \\
& \leqslant C\left|a_{1}(x)\right| \int_{1}^{\eta / x}(\lambda x)^{\alpha+3 / 2} \frac{1}{\lambda} d \lambda+C\left|a_{1}(t)\right| \int_{1}^{\eta / x}(\lambda x)^{\alpha+1 / 2} \frac{1}{\lambda} d \lambda \leqslant C .
\end{aligned}
$$


This proves the inequality when $x \leqslant \eta R^{-1}$. When $x \geqslant \eta R^{-1}$, all that is left to show is the boundedness of

$$
\left|\int_{\eta / x}^{R}\left(a_{1}(x) \mathcal{J}_{\alpha+1}(\lambda x) \mathcal{J}_{\alpha}(\lambda t)+a_{1}(t) \mathcal{J}_{\alpha+1}(\lambda t) \mathcal{J}_{\alpha}(\lambda x)\right) \frac{1}{\lambda} d \lambda\right|
$$

Using the classical asymptotic expansion for $\mathcal{J}_{\alpha}(z)$

$$
\mathcal{J}_{\alpha}(z)=\sqrt{\frac{2}{\pi}} \cos \left(z-\gamma_{\alpha}\right)+O\left(z^{-1}\right)
$$

for $z \rightarrow \infty$ (here $\left.\gamma_{\alpha}=(2 \alpha+1) \pi / 4\right)$ it is not difficult to reduce the boundedness of the above integral to the boundedness of

$$
\int_{\eta / x}^{R} a_{1}(x) \cos \left(\lambda x-\gamma_{\alpha+1}\right) \cos \left(\lambda t-\gamma_{\alpha}\right)+a_{1}(t) \cos \left(\lambda t-\gamma_{\alpha+1}\right) \cos \left(\lambda x-\gamma_{\alpha}\right) \frac{d \lambda}{\lambda} .
$$

Since

$$
\begin{aligned}
& a_{1}(x) \cos \left(\lambda x-\gamma_{\alpha+1}\right) \cos \left(\lambda t-\gamma_{\alpha}\right)+a_{1}(t) \cos \left(\lambda t-\gamma_{\alpha+1}\right) \cos \left(\lambda x-\gamma_{\alpha}\right) \\
& =\frac{1}{2} a_{1}(x)\left[\cos (\lambda(x+t)-(\alpha+1) \pi)+\cos \left(\lambda(x-t)-\frac{\pi}{2}\right)\right] \\
& \quad+\frac{1}{2} a_{1}(t)\left[\cos (\lambda(x+t)-(\alpha+1) \pi)+\cos \left(\lambda(t-x)-\frac{\pi}{2}\right)\right] \\
& =\frac{1}{2}\left(a_{1}(x)+a_{1}(t)\right) \cos (\lambda(x+t)-(\alpha+1) \pi) \\
& +\frac{1}{2}\left[a_{1}(t)-a_{1}(x)\right] \sin (\lambda(t-x))
\end{aligned}
$$

we have

$$
\begin{aligned}
& \int_{\eta / x}^{R} a_{1}(x) \cos \left(\lambda x-\gamma_{\alpha+1}\right) \cos \left(\lambda t-\gamma_{\alpha}\right)+a_{1}(t) \cos \left(\lambda t-\gamma_{\alpha+1}\right) \cos \left(\lambda x-\gamma_{\alpha}\right) \frac{d \lambda}{\lambda} \\
& =\int_{\eta / x}^{R} \frac{1}{2}\left[a_{1}(x)+a_{1}(t)\right] \cos (\lambda(x+t)-(\alpha+1) \pi) \frac{d \lambda}{\lambda} \\
& \quad+\int_{\eta / x}^{R} \frac{1}{2}\left[a_{1}(t)-a_{1}(x)\right] \sin (\lambda(t-x)) \frac{d \lambda}{\lambda} .
\end{aligned}
$$

Integrating by parts and using the fact that $a_{1}$ is $C^{1}$ and satisfies $\left|a_{1}(t)\right| \leqslant C t$ gives the desired result. 
Proof of Theorem 0.2. By (3.1) and (3.2) the theorem is a simple consequence of the fact that for $f \in L^{1}\left(\frac{1}{1+t} d t\right), \widetilde{F}_{R} f$ and $\widetilde{S}_{R} f$ are well defined and

$$
\lim _{R \rightarrow+\infty}\left[\widetilde{S}_{R} f(x)-\widetilde{F}_{R} f(x)\right]=0
$$

To see this observe that $\widetilde{F}_{R} f$ is well defined by Theorem 0.1 , and that

$$
\begin{aligned}
\left|\widetilde{S}_{R} f(x)-\widetilde{F}_{R} f(x)\right| & \leqslant \int_{0}^{+\infty}\left|\widetilde{S}_{R}(x, t)-\widetilde{F}_{R}(x, t)\right||f(t)| d t \\
& =\int_{0}^{+\infty}(1+t)\left|\widetilde{S}_{R}(x, t)-\widetilde{F}_{R}(x, t)\right| \frac{|f(t)|}{1+t} d t .
\end{aligned}
$$

For every fixed $x \geqslant 0$, by the previous lemma $(1+t)\left|\widetilde{S}_{R}(x, t)-\widetilde{F}_{R}(x, t)\right|$ is bounded in $t$. For every fixed $x \geqslant 0$ and for every $t>0$

$$
\lim _{R \rightarrow+\infty}(1+t)\left|\widetilde{S}_{R}(x, t)-\widetilde{F}_{R}(x, t)\right|=0,
$$

and by the dominated convergence theorem (3.3) follows.

Proof of Corollary 0.3. Let $f \in L^{q}(A(t) d t)$. It is easy to check that

$$
f \in L^{1}\left(\frac{\sqrt{A(t)}}{1+t} d t\right) .
$$

By Theorem 0.2 and Theorem $0.1, S_{R} f(x)$ is equiconvergent with

$$
\frac{1}{\sqrt{A(x)}} C_{R}(\sqrt{A} f)(x) \text {. }
$$

Since for every $0<\varepsilon<\eta$ we have $\sqrt{A} f \in L^{q}([\varepsilon, \eta], d t)$, by the localization principle and the Carleson-Hunt theorem we have that $\frac{1}{\sqrt{A(x)}} C_{R}(\sqrt{A} f)(x)$ converges almost everywhere to $f(x)$ in $[\varepsilon, \eta]$ and so does $S_{R} f(x)$.

Proof of Theorem 0.4. Assume $\rho>0$ and let $g \in L^{q}(A(t) d t)$ with $q<2$. Using the asymptotic expansion for $\Phi_{\lambda}$ and $c(\lambda)$ one can easily check that $\varphi_{\lambda}=$ $c(\lambda) \Phi_{\lambda}+c(-\lambda) \Phi_{-\lambda} \in L^{r}(A(t) d t)$ for any $r>2$. It follows that $\widehat{g}$ is continuous. Assume now that for some $p>2, S_{R}$ maps $L^{p}(A(t) d t)$ into tempered distributions. By duality $S_{R}$ maps test functions in $L^{p^{\prime}}(A(t) d t)$. Since $p^{\prime}<2$ it follows that for every test function $f, \widehat{S_{R} f}$ is continuous. However the $L^{2}$ theory for the Fourier transform (see e.g. [1, Chapter 2]) shows that $\widehat{S_{R} f}=\chi_{[0, R]} \widehat{f}$.

Let now $\rho=0$ and assume that $S_{R}$ maps $L^{p}(A(x) d x)$ into tempered distribution for some $p \geqslant \frac{4 b+4}{2 b+1}$. By duality $S_{R}$ maps test functions in $L^{q}(A(x) d x)$ for 
some $q \leqslant \frac{4 b+4}{2 b+3}$. Let $f \not \equiv 0$ be smooth, even and compactly supported. Let $R$ such that $\widehat{f}(R) \neq 0$. We have

$$
\begin{aligned}
S_{R} f(x) & =\int_{0}^{R} \widehat{f}(R) \varphi_{\lambda}(x) \frac{d \lambda}{2 \pi|c(\lambda)|^{2}}+\int_{0}^{R}[\widehat{f}(\lambda)-\widehat{f}(R)] \varphi_{\lambda}(x) \frac{d \lambda}{2 \pi|c(\lambda)|^{2}} \\
& =I+I I .
\end{aligned}
$$

A change in the path of integration in $I$ (as in the proof of Lemma 3.1) and (2.1) give

$$
I=\frac{\widehat{f}(R)}{2 \pi} \int_{\gamma} \Phi_{-\lambda}(x) \frac{d \lambda}{c(\lambda)}
$$

where $\gamma=\left\{\operatorname{Re}^{i \theta}:-\pi \leqslant \theta \leqslant 0\right\}$. By Theorem 1.17 we have

$$
\Phi_{-\lambda}(x)=A^{-\frac{1}{2}}(x) e^{-i \lambda x}(1+\mathcal{R}(\lambda, x))
$$

with

$$
|\mathcal{R}(\lambda, x)| \leqslant e^{\frac{1}{|\lambda|} \int_{x}^{+\infty}|q(v)| d v}-1 .
$$

By (H5), for large values of $\lambda$ and $x$ we obtain

$$
\Phi_{-\lambda}(x)=A^{-\frac{1}{2}}(x) e^{-i \lambda x}\left(1+O\left(\frac{1}{\lambda x}\right)\right) .
$$

By Corollary 2.3,

$$
\begin{aligned}
I & =\frac{\widehat{f}(R)}{2 \pi} \int_{\gamma} A^{-\frac{1}{2}}(x) e^{-i \lambda x} \frac{d \lambda}{c(\lambda)}+\frac{\widehat{f}(R)}{2 \pi} \int_{\gamma} A^{-\frac{1}{2}}(x) e^{-i \lambda x} O\left(\frac{1}{\lambda x}\right) \frac{d \lambda}{c(\lambda)} \\
& =\frac{\widehat{f}(R)}{2 \pi} A^{-\frac{1}{2}}(x) \int_{\gamma} e^{-i \lambda x} \frac{d \lambda}{c(\lambda)}+O\left(\widehat{f}(R) A^{-\frac{1}{2}}(x) \int_{-\pi}^{0} e^{R x \sin \theta} \frac{1}{R x} R^{\alpha+\frac{1}{2}} R d \theta\right) \\
& =\frac{\widehat{f}(R)}{2 \pi} A^{-\frac{1}{2}}(x) \int_{\gamma} e^{-i \lambda x} \frac{d \lambda}{c(\lambda)}+O\left(\widehat{f}(R) \frac{A^{-\frac{1}{2}}(x) R^{\alpha-\frac{1}{2}}}{x^{2}}\right) .
\end{aligned}
$$

Also, by Theorem 2.1

$$
\frac{1}{c(\lambda)}=c \lambda^{\alpha+\frac{1}{2}}+O\left(\lambda^{\alpha-\frac{1}{2}}\right)
$$

and therefore

$$
\begin{aligned}
\int_{\gamma} e^{-i \lambda x} \frac{d \lambda}{c(\lambda)} & =c \int_{\gamma} e^{-i \lambda x} \lambda^{\alpha+\frac{1}{2}} d \lambda+O\left(\int_{\gamma} e^{-i \lambda x} \lambda^{\alpha-\frac{1}{2}} d \lambda\right) \\
& =c \int_{-R}^{R} e^{-i \lambda x} \lambda^{\alpha+\frac{1}{2}} d \lambda+O\left(\frac{R^{\alpha-\frac{1}{2}}}{x}\right) .
\end{aligned}
$$


Some standard computations show that

$$
\int_{-R}^{R} e^{-i \lambda x} \lambda^{\alpha+\frac{1}{2}} d \lambda=c \sin \left(R x-\frac{\pi}{2}\left(\alpha+\frac{1}{2}\right)\right) \frac{R^{\alpha+\frac{1}{2}}}{x}+O\left(\frac{R^{\alpha-\frac{1}{2}}}{x^{2}}\right)
$$

and therefore

$$
I=c \widehat{f}(R) A^{-\frac{1}{2}}(x) \frac{R^{\alpha+\frac{1}{2}}}{x}\left[\sin \left(R x-\frac{\pi}{2}\left(\alpha+\frac{1}{2}\right)\right)+O\left(\frac{1}{R}\right)\right] .
$$

Let now $x$ such that $\left|\sin \left(R x-\frac{\pi}{2}\left(\alpha+\frac{1}{2}\right)\right)\right|>\frac{1}{2}$ and $R$ sufficiently large such that $O\left(\frac{1}{R}\right)<\frac{1}{4}$. We have

$$
I>c \widehat{f}(R) A^{-\frac{1}{2}}(x) \frac{R^{\alpha+\frac{1}{2}}}{x} .
$$

We now estimate $I I$. A similar computation shows that

$$
\begin{aligned}
|I I| & =\left|\frac{1}{2 \pi} \int_{\gamma}[\widehat{f}(\lambda)-\widehat{f}(R)] \Phi_{-\lambda}(x) \frac{d \lambda}{c(\lambda)}\right| \\
& \leqslant c \int_{0}^{\pi}\left|\widehat{f}\left(R e^{-i R \theta}\right)-\widehat{f}(R)\right| A^{-\frac{1}{2}}(x) e^{-R x \sin \theta} R^{\alpha+\frac{3}{2}} d \theta .
\end{aligned}
$$

Since $\widehat{f}\left(R e^{-i R \theta}\right)-\widehat{f}(R)=0$ for $\theta=0$ and $\theta=\pi$ it is easy to see that

$$
|I I| \leqslant c_{R} \frac{A^{-\frac{1}{2}}(x)}{x^{2}} .
$$

Let now $x$ such that $\left|\sin \left(R x-\frac{\pi}{2}\left(\alpha+\frac{1}{2}\right)\right)\right|>\frac{1}{2}$ then

$$
\begin{aligned}
\left|S_{R} f(x)\right| & =|I+I I| \geqslant c \widehat{f}(R) A^{-\frac{1}{2}}(x) \frac{R^{\alpha+\frac{1}{2}}}{x}-c_{R} \frac{A^{-\frac{1}{2}}(x)}{x^{2}} \\
& =\frac{A^{-\frac{1}{2}}(x)}{x}\left(c \widehat{f}(R) R^{\alpha+\frac{1}{2}}-c_{R} \frac{1}{x}\right) .
\end{aligned}
$$

Since $A(x) \approx x^{2 b+1}\left(A(x) \approx x \log ^{2} x\right.$ in the singular case), integrating over $\left\{x:\left|\sin \left(R x-\frac{\pi}{2}\left(\alpha+\frac{1}{2}\right)\right)\right|>\frac{1}{2}\right\}$ shows that $S_{R} f(x) \notin L^{q}(A(x) d x)$ for any $q \leqslant \frac{4 b+4}{2 b+3}$ (observe that since $\widehat{f}$ is entire we can always find large values of $R$ such that $\widehat{f}(R) \neq 0)$.

Proof of Theorem 0.5. In the case of the Fourier-Bessel expansion the result is contained in [6]. Using the asymptotic expansion for $\varphi_{\lambda}$ given Theorem 1.2 and the estimate for $|c(\lambda)|$ given in Corollary 2.3 one can easily adapt their proof. 


\section{A. Appendix}

In this appendix we collect some known facts used repeatedly in the paper.

Lemma A.1. Let $u_{1}$ and $u_{2}$ be two linearly independent solutions of the equation

$$
u^{\prime \prime}+p_{1} u^{\prime}+p_{2} u=0 .
$$

If $\varphi(t)$ is a $\mathcal{C}^{2}$ solution of the integral equation

$$
u(t)=-\int_{t_{0}}^{t} \frac{u_{1}(t) u_{2}(s)-u_{2}(t) u_{1}(s)}{u_{1}(s) u_{2}^{\prime}(s)-u_{2}(s) u_{1}^{\prime}(s)}\left(\psi_{0}(s) u(s)+J(s) \phi(s)\right) d s,
$$

then $\varphi(t)$ is a solution of the differential equation

$$
u^{\prime \prime}+p_{1} u^{\prime}+p_{2} u=\psi_{0} u+J \phi .
$$

The above integral equation can be solved by the method of successive approximation. For reader's convenience, we restate of [11, Theorem 10.1, Chapter VI].

Let us consider a kernel $k(t, s)$ defined for $t \in\left(t_{0}, t_{1}\right)$ and $s \in\left(t_{0}, t\right]$ that satisfies the following conditions

1. $k(t, s)$ and $\frac{\partial k}{\partial t}(t, s)$ are continuous,

2. for $t \in\left(t_{0}, t_{1}\right)$ we have $k(t, t)=0$,

3. there exist continuous functions $P_{0}, P_{1}$ and $Q$ such that, for all $s \in\left(t_{0}, t\right]$,

$$
|k(t, s)| \leqslant P_{0}(t) Q(s)
$$

and

$$
\left|\frac{\partial k}{\partial t}(t, s)\right| \leqslant P_{1}(t) Q(s),
$$

4.

$$
\kappa=\sup _{t \in\left(t_{0}, t_{1}\right)} Q(t)|J(t)|<+\infty
$$

and

$$
\kappa_{0}=\sup _{t \in\left(t_{0}, t_{1}\right)} Q(t) P_{0}(t)<+\infty .
$$

Theorem A.2. Given an integral equation of the form

$$
u(t)=-\int_{t_{0}}^{t} k(t, s)\left(\psi_{0}(s) u(s)+J(s) \phi(s)\right) d s
$$

assume that

1. the functions $\psi_{0}, J$, and $\phi$ are continuous,

2. the following integrals converge

$$
\int_{t_{0}}^{t}|\phi(s)| d s, \quad \int_{t_{0}}^{t}\left|\psi_{0}(s)\right| d s,
$$


then (A.1) has a unique twice continuously differentiable solution $u(t)$ satisfying

$$
\frac{u(t)}{P_{0}(t)} \rightarrow 0, \quad \frac{u^{\prime}(t)}{P_{1}(t)} \rightarrow 0 \quad \text { as } t \rightarrow t_{0}
$$

Furthermore

$$
\frac{|u(t)|}{P_{0}(t)}, \frac{\left|u^{\prime}(t)\right|}{P_{1}(t)} \leqslant \kappa\left|\int_{t_{0}}^{t}\right| \phi(s)|d s| \exp \left(\kappa_{0}\left|\int_{t_{0}}^{t}\right| \psi_{0}(s)|d s|\right) .
$$

If $\phi(s)=\psi_{0}(s)$, then the above estimate can be sharpened

$$
\frac{|u(t)|}{P_{0}(t)}, \frac{\left|u^{\prime}(t)\right|}{P_{1}(t)} \leqslant \frac{\kappa}{\kappa_{0}}\left(\exp \left(\kappa_{0}\left|\int_{t_{0}}^{t}\right| \phi(s)|d s|\right)-1\right) .
$$

Theorem A.3. Let $k$ as above and assume that $J$ is differentiable on $\left(t_{0}, t_{1}\right)$ and $\phi(s)$ continuous on $\left(t_{0}, t_{1}\right)$, such that $\int_{t_{0}}^{t}|\phi(s)| d s$ converges for every $t \in\left(t_{0}, t_{1}\right)$. Let $u_{0} \equiv 0$ and define inductively

$$
u_{j+1}(t)=J(t)-\int_{t_{0}}^{t} k(t, s) \phi(s) u_{j}(s) d s .
$$

Then

$$
\left|u_{j+1}(t)-u_{j}(t)\right| \leqslant \frac{\kappa}{\kappa_{0}} P_{0}(t) \frac{\left(\kappa_{0} \int_{t_{0}}^{t}|\phi(s)| d s\right)^{j+1}}{j !}
$$

and

$$
\left|u_{j+1}^{\prime}(t)-u_{j}^{\prime}(t)\right| \leqslant \frac{\kappa}{\kappa_{0}} P_{1}(t) \frac{\left(\kappa_{0} \int_{t_{0}}^{t}|\phi(s)| d s\right)^{j+1}}{j !} .
$$

Moreover

$$
u(t)=\sum_{j=0}^{+\infty}\left(u_{j+1}(t)-u_{j}(t)\right)
$$

is the unique solution of the integral equation

$$
u(t)=J(t)-\int_{t_{0}}^{t} k(t, s) \phi(s) u(s) d s
$$

such that

$$
\frac{u(t)-J(t)}{P_{0}(t)} \rightarrow 0, \quad \frac{u^{\prime}(t)-J^{\prime}(t)}{P_{1}(t)} \rightarrow 0 \quad \text { as } t \rightarrow t_{0}
$$


Furthermore

$$
\begin{gathered}
|u(t)-J(t)| \leqslant P_{0}(t) \frac{\kappa}{\kappa_{0}}\left(\exp \left(\kappa_{0}\left|\int_{t_{0}}^{t}\right| \phi(s)|d s|\right)-1\right) \\
\left|u^{\prime}(t)-J^{\prime}(t)\right| \leqslant P_{1}(t) \frac{\kappa}{\kappa_{0}}\left(\exp \left(\kappa_{0}\left|\int_{t_{0}}^{t}\right| \phi(s)|d s|\right)-1\right) .
\end{gathered}
$$

\section{References}

[1] W. R. Bloom and H. Heyer, "Harmonic Analysis of Probability Measures on Hypergroups", de Gruyter Studies in Mathematics, Vol. 20, 1994.

[2] W. R. BLOOM and Z. XU, The Hardy-Littlewood maximal function for Chébli-Trimèche hypergroups, Contemp. Math. 183 (1995), 45-70.

[3] M. BôCHER, On regular singular points of linear differential equations of the second order whose coefficients are not necessarily analytic, Trans. Amer. Math. Soc. 1 (1990), 40-52.

[4] O. Bracco, "Fonction Maximale Associée à des Opérateurs de Sturm-Liouville Singuliers", Thèse, Université Louis Pasteur, Strasbourg, 1999.

[5] H. Chebli, Sur un Théorème de Paley-Wiener Associé à la Décomposition Spectrale d'un Opérateur de Sturm-Liouville sur ]0, $+\infty$ [, J. Funct. Anal. 17 (1974), 447-461.

[6] L. Colzani, A. Crespi, G. Travaglini and M. Vignati, Equiconvergence theorems for Fourier-Bessel expansions with applications to the harmonic analysis of radial functions in Euclidean and non-Euclidean spaces, Trans. Amer. Math. Soc. 338 (1993), 43-55.

[7] A. Fitouni and M. M. HAMZA, A uniform expansion for the eigenfunction of a singular second-order differential operator, SIAM J. Math Anal. 21 (1990), 1619-1632.

[8] M. FLENSTED-JENSEN and T. KOORNWINDER, The convolution structure for Jacobi function expansions, Ark. Mat. 11 (1973), 245-262.

[9] L. Gallardo and K. TRIMÈCHe, Renewal theorems for Singular Differential Operators, J. Theoret. Probab. 15 (2002), 161-205.

[10] N. N. LebedEV, "Special Functions and their Applications", Dover Publications, 1972.

[11] F. W. J. Olver, "Asymptotic and Special function", AKP Classics, 1997.

[12] C. MeAney and E. PREstini, Almost everywhere convergence of inverse spherical transforms on noncompact symmetric spaces, J. Funct. Anal. 149 (1997), 277-304.

[13] C. MeAney and E. PRestini, On almost-everywhere convergence of inverse spherical transforms, Pacific J. Math. 170 (1995), 203-215.

[14] R. J. STANTON and P. A. TOMAS, Expansions for spherical functions on noncompact symmetric spaces, Acta Math. 140 (1978), 251-276.

[15] G. N. WATSON, "A treatise on the Theory of Bessel Functions", 2nd edition, Cambridge, University Press, 1996.

[16] H. ZeUneR, One dimensional hypergroup, Adv. Math. 76 (1989), 1-18.

Dipartimento di Ingegneria dell'Informazione e Metodi Matematici

Università degli Studi di Bergamo

Viale Marconi, 5

24044 Dalmine, Italia

luca.brandolini@unibg.it

giacomo.gigante@unibg.it 